\title{
Cooperative Localization in WSNs Using Gaussian Mixture Modeling: Distributed ECM Algorithms
}

\author{
Feng Yin, Carsten Fritsche, Di Jin, Fredrik Gustafsson and Abdelhak M. Zoubir
}

\section{Linköping University Post Print}

\section{Tweet}

N.B.: When citing this work, cite the original article.

Feng Yin, Carsten Fritsche, Di Jin, Fredrik Gustafsson and Abdelhak M. Zoubir, Cooperative Localization in WSNs Using Gaussian Mixture Modeling: Distributed ECM Algorithms, 2015, IEEE Transactions on Signal Processing, (63), 6, 1448-1463.

http://dx.doi.org/10.1109/TSP.2015.2394300

C2015 IEEE. Personal use of this material is permitted. However, permission to reprint/republish this material for advertising or promotional purposes or for creating new collective works for resale or redistribution to servers or lists, or to reuse any copyrighted component of this work in other works must be obtained from the IEEE.

\section{http://ieeexplore.ieee.org/}

Postprint available at: Linköping University Electronic Press

http://urn.kb.se/resolve?urn=urn:nbn:se:liu:diva-116504 


\title{
Cooperative Localization in WSNs Using Gaussian Mixture Modeling: Distributed ECM Algorithms
}

\author{
Feng Yin, Member, IEEE, Carsten Fritsche, Member, IEEE, Di Jin, Student Member, IEEE, \\ Fredrik Gustafsson, Fellow, IEEE and Abdelhak M. Zoubir, Fellow, IEEE
}

\begin{abstract}
We study cooperative sensor network localization in a realistic scenario where (1) the underlying measurement errors more probably follow a non-Gaussian distribution; (2) the measurement error distribution is unknown without conducting massive offline calibrations; and (3) non-line-of-sight identification is not performed due to the complexity constraint and/or storage limitation. The underlying measurement error distribution is approximated parametrically by a Gaussian mixture with finite number of components, and the expectation-conditional maximization (ECM) criterion is adopted to approximate the maximum-likelihood estimator of the unknown sensor positions and an extra set of Gaussian mixture model parameters. The resulting centralized ECM algorithms lead to easier inference tasks and meanwhile retain several convergence properties with a proof of the "space filling" condition. To meet the scalability requirement, we further develop two distributed ECM algorithms where an average consensus algorithm plays an important role for updating the Gaussian mixture model parameters locally. The proposed algorithms are analyzed systematically in terms of computational complexity and communication overhead. Various computer based tests are also conducted with both simulation and experimental data. The results pin down that the proposed distributed algorithms can provide overall good performance for the assumed scenario even under model mismatch, while the existing competing algorithms either cannot work without the prior knowledge of the measurement error statistics or merely provide degraded localization performance when the measurement error is clearly non-Gaussian.
\end{abstract}

Index Terms-Centralized and distributed algorithms, cooperative localization, expectation-conditional maximization (ECM), Gaussian mixture, wireless sensor network (WSN).

\section{INTRODUCTION}

The use of wireless sensor networks (WSNs), consisting of lightweight, low-cost, low-power, multi-functional sensors, has driven a myriad of monitoring, tracking, and control applications [1]. Often, the data collected by a sensor node must be tied with the sensor position in order to be meaningful. In WSNs, in general, only a few sensor nodes, called "anchors" in the sequel, know their positions a priori, while

Copyright (c) 2014 IEEE. Personal use of this material is permitted. However, permission to use this material for any other purposes must be obtained from the IEEE by sending a request to pubs-permissions@ieee.org.

F. Yin was with the Signal Processing Group, Institute of Telecommunications, Technische Universität Darmstadt, Darmstadt, 64283, Germany. $\mathrm{He}$ is now with Ericsson Research, Linköping, SE-58330, Sweden (e-mail: fyin@spg.tu-darmstadt.de or feng.yin@ericsson.com)

D. Jin and A. M. Zoubir are with the Signal Processing Group, Institute of Telecommunications, Technische Universität Darmstadt, Darmstadt, 64283, Germany (e-mail: djin@spg.tu-darmstadt.de; zoubir@spg.tu-darmstadt.de).

C. Fritsche and F. Gustafsson are with the Department of Electrical Engineering, Division of Automatic Control, Linköping University, Linköping, SE-58183, Sweden (e-mail: carsten@isy.liu.se; fredrik@isy.liu.se). the remaining sensor nodes with unknown positions, called "agents" in the sequel, need to be localized [2]. Cooperation is an emerging paradigm for sensor network localization, which exploits additionally the inter-agent measurements and as a consequence enables more robust and accurate inference of the unknown positions.

In the past decade, a plethora of cooperative localization algorithms has been proposed based on different position related signal metrics, such as received-signal-strength (RSS), time-of-arrival (TOA), and round-trip TOA (RTOA). They can be broadly categorized into non-Bayesian algorithms and Bayesian algorithms. In the non-Bayesian algorithms, the unknown positions are assumed to be deterministic. Classical non-Bayesian algorithms (both centralized and distributed) include: (1) least-squares (LS) estimation based algorithms [3], [4], and [5, Algorithm 1]; (2) multi-dimensional scaling (MDS) based algorithms [6], [8]; (3) programming based algorithms [9], [10]; (4) iterative parallel projection method (IPPM) based algorithms [11], [12]. In the Bayesian algorithms, unknown positions are assumed to be random variables with a certain prior distribution. Representative Bayesian algorithms include the nonparametric belief propagation (NBP) algorithm [13], the sum-product algorithm over a wireless network, short for SPAWN algorithm [5] in the sequel, and several new variations [14]-[17] built upon them, which perform message passing by taking advantages of the belief propagation algorithm [18] or the sum-product algorithm [19] in different graphical models.

Restricted by the ad-hoc nature of a WSN, distributed cooperative localization (also known as self-localization) algorithms are highly sought for. This is owing to their advantageous features of being scalable, independent of fusion center, and less sensitive to sensor failure as compared to the centralized solutions [20]. In the distributed non-Bayesian algorithms, wireless sensors exchange their position estimates mutually; while in the conventional, distributed Bayesian algorithms, they exchange local belief messages (distributions represented by a set of particles) about their actual positions and thus consume much more energy for communication. At the sacrifice of localization accuracy, however, recent work demonstrated that the communication overhead can be significantly reduced by using transmit- and receive censoring [17] and/or parametric representation of the local belief messages [16], [21]. As compared to non-Bayesian algorithms, Bayesian algorithms for localization purposes may include prior information about sensor positions, sensor movement, noise statistics and so on, thus offering a greater flexibility such that robustness consid- 
erations can be accounted for. However, even though Bayesian approaches might be superior to non-Bayesian ones, it is often difficult to adequately choose the prior, and a wrongly chosen prior may severely deteriorate the performance.

Unlike the assumption made in a majority of the existing algorithms, the ranging measurement error was found to deviate from a single Gaussian model in various measurement campaigns, see for instance [5], [22]-[24] for both cooperative and non-cooperative localization. In this paper, we approximate the unknown underlying measurement error distribution by a Gaussian mixture despite model mismatch. To be realistic, we assume that the mixture model parameters are unknown. Our goal is to approximate the maximum likelihood estimator (MLE) of the unknown positions and the Gaussian mixture parameters with lower computational complexity and less communication overhead.

The original contributions of this paper are as follows. We develop a series of centralized and distributed expectationconditional maximization (ECM) algorithms (non-Bayesian in nature) based on distance measurements (may be converted from TOA or RTOA measurements) to approximate the MLE of the unknown parameters. The proposed algorithms are evaluated in terms of computational complexity and communication overhead and further compared with several competing algorithms comprehensively in terms of the localization accuracy. More emphasis is laid on the distributed ECM algorithms. Lastly, we want to mention that this paper is built on our previous work in [25], where a centralized algorithm was proposed for cooperative localization. As will be explained later, this algorithm is also an ECM algorithm but can be numerically unstable as compared to two other examples for very large-scale WSNs. Besides, decentralization of this algorithm is infeasible.

The remainder of this paper is organized as follows. Section II introduces the signal model and states the problem at hand. Section III introduces a series of ECM algorithms to approximate the MLE with less computational efforts. Section IV elaborates on the computational complexity and energy consumption of the proposed ECM algorithms. In Section V, we perform Cramér-Rao bound (CRB) analysis and show the best achievable localization accuracy, followed by a comprehensive simulation in Section VI. Finally, Section VII concludes the paper.

Notation: Throughout this paper, matrices are presented with boldface uppercase letters and vectors with boldface lowercase letters. $\mathbb{Z}$ denotes the set of integers. The operator $[\cdot]^{T}$ stands for vector/matrix transpose and $\operatorname{tr}[\cdot]$ denotes the trace of a square matrix. $\|\cdot\|$ stands for the Euclidean norm for a vector and $|\cdot|$ denotes the cardinality of a set. $\mathbf{X} \succeq \mathbf{Y}$ means that the matrix difference $\mathbf{X}-\mathbf{Y}$ is positive semidefinite. The operator $\mathbb{E}_{p(x)}(\cdot)$ stands for the expectation taken with respect to the probability density function (PDF) $p(x)$. Further, $\nabla_{\boldsymbol{\theta}}=\partial / \partial \boldsymbol{\theta}$ denotes the gradient operator and $\Delta_{\boldsymbol{\theta}}^{\boldsymbol{\theta}}=\nabla_{\boldsymbol{\theta}} \nabla_{\boldsymbol{\theta}}^{T}$ denotes the Laplace operator. $\mathcal{N}\left(v ; \mu, \sigma^{2}\right)$ denotes the Gaussian distribution of a random variable $V$ with mean $\mu$ and variance $\sigma^{2}$, and $\mathcal{U}[a, b)$ denotes the uniform distribution on the interval $[a, b)$ with $a<b$.

\section{Signal Model and Problem Formulation}

\section{A. Signal Model}

We consider cooperative localization in a connected network comprising a total number of $N$ wireless sensors in a two-dimensional (2-D) space (although extension to the 3-D case is straightforward). We let, without loss of generality, $\mathcal{N}_{a}=\left\{1,2, \ldots, N_{u}\right\}$ be the set of indices of the agents, whose positions $\left\{\boldsymbol{p}_{i}=\left[x_{i}, y_{i}\right]^{T}, i \in \mathcal{N}_{a}\right\}$ are unknown, and let $\mathcal{N}_{b}=\left\{N_{u}+1, N_{u}+2, \ldots, N\right\}$ be the set of indices of the anchors with known positions.

In order to localize the agents, we adopt a two-stage procedure. In the first stage, every sensor broadcasts its sensor ID and listens for its neighboring sensors' broadcasts. Then, each agent obtains a set of distance measurements relative to its neighboring sensors, which can be done, for instance, by estimating the TOA of a received signal. Note that nonline-of-sight (NLOS) identification can be done in the first stage. However, we avoid it in this paper as low-complexity and low-cost algorithms are sought for. In the second stage, the unknown agent positions will be estimated concurrently in a localization algorithm based on the obtained distance measurements. There can be another initialization stage in between, if no empirical knowledge about the agent positions and the environment is available.

We consider a statistical measurement model

$$
r_{i, j}=d\left(\boldsymbol{p}_{i}, \boldsymbol{p}_{j}\right)+v_{i, j}
$$

where $r_{i, j}$ is a distance measurement obtained at sensor $i$ in communication with sensor $j, d\left(\boldsymbol{p}_{i}, \boldsymbol{p}_{j}\right)=\left\|\boldsymbol{p}_{i}-\boldsymbol{p}_{j}\right\|$ is the true Euclidean distance between the two sensors, and $v_{i, j}$ is an additive measurement error. The main sources of error include thermal noise, multiple access interference, multi-path effects, (TOA estimation) algorithm artifacts, and possibly also the NLOS propagation. The measurement errors for different sensor pairs are assumed to be independently and identically distributed (i.i.d.) according to $p_{V}(v)$, which is approximated by a Gaussian mixture with finite number of components, namely,

$$
p_{V}(v) \approx \sum_{l=1}^{C} \alpha_{l} \mathcal{N}\left(v ; \mu_{l}, \sigma_{l}^{2}\right)
$$

where $C$ is the total number of mixture components, $\alpha_{l}$ is the mixing coefficient of the lth Gaussian component $\mathcal{N}\left(v ; \mu_{l}, \sigma_{l}^{2}\right)$. The mixture model parameters $\alpha_{l}, \mu_{l}, \sigma_{l}^{2}, l=$ $1,2, \ldots, C$, are assumed to be unknown. The motivations are twofold. On the one hand, the underlying measurement error statistics may vary with both the localization scenario due to the change of obstructions and time due to the dynamic nature of the environment, and we cannot afford repetitive massive offline calibrations; On the other hand, offline calibration is hard to conduct in inaccessible or unexplored environments, even if the measurement error statistics can be quasi-static.

The model in (2) is popular for representing noise in various signal processing applications. This is because any distribution can be approximated as closely as desired in $\mathcal{L}_{1}$ norm by a Gaussian mixture [26]. Even if (2) should deviate from the underlying measurement error model for small $C$ in most 
cases, it is still capable of capturing some key features of the measurement errors. The contaminated Gaussian model, famous for its robustness against outliers from the robust statistics [27], is a special case of (2) with $C=2$ and $\mu_{1}=\mu_{2}=0$. Lastly, we note that the i.i.d. assumption on the measurement errors may not always hold in practice but allows for better mathematical tractability.

\section{B. Problem Formulation}

We propose to jointly estimate the unknown deterministic positions $\left\{\boldsymbol{p}_{i}, i \in \mathcal{N}_{a}\right\}$ and the mixture model parameters $\alpha_{l}$, $\mu_{l}, \sigma_{l}^{2}, l=1, \ldots, C$, given the statistical measurement model (1), a set of noisy distance measurements $\left\{r_{i, j}\right\}$, and a few known anchor positions $\left\{\boldsymbol{p}_{i}, i \in \mathcal{N}_{b}\right\}$.

\section{MAXimum Likelihood Estimation}

In Section III-A, we briefly comment on the difficulties with the conventional maximum-likelihood implementation. To approximate the MLE with less computational hurdles, we develop a series of expectation-conditional maximization (ECM) algorithms (cf. Section III-B for centralized solutions and Section III-C for distributed solutions). Section III-D gives a practical initialization strategy, and Section III-E introduces a possible extension.

\section{A. Maximum Likelihood Estimation}

We start with the conventional implementation (centralized in nature) of the maximum likelihood estimation. As we know, in centralized algorithms all the measurements have to be transmitted via multi-hop routing to a fusion center for processing. In order to reduce the communication overhead and computational complexity, like the two representative algorithms [3], [8], we assume $r_{i, j}=r_{j, i}$ and only one of them (e.g., choose $r_{i, j}$ with $j>i$ ) will be routed to the fusion center throughout this paper. This assumption approximately holds when the sensors are equipped with an omni-directional antenna and conduct the interrogation in a pair of reciprocal channels. Besides, distance measurements obtained between anchors are ignored when estimating the agent positions because they are irrelevant. But they are useful for the initialization of the Gaussian mixture model parameters, as will be further elaborated in Section III-D. In this way, only c.a. half of the measurements will be used to make an inference in the fusion center. Before proceeding further, we introduce the following notations:

- $\boldsymbol{\theta}=\left[\boldsymbol{\theta}_{m}^{T}, \boldsymbol{\theta}_{p}^{T}\right]^{T}$ is a vector of all unknown parameters, where $\boldsymbol{\theta}_{m}=\left[\alpha_{1}, \alpha_{2}, \ldots, \alpha_{C}, \mu_{1}, \sigma_{1}^{2}, \mu_{2}, \sigma_{2}^{2}, \ldots, \mu_{C}, \sigma_{C}^{2}\right]^{T}$ and $\boldsymbol{\theta}_{p}=\left[\boldsymbol{p}_{1}^{T}, \ldots, \boldsymbol{p}_{N_{u}}^{T}\right]^{T}$.

- $R_{c}$ is the maximal communication range of a sensor. An ideal model is adopted for determining the neighborhood of each agent throughout this paper. In practice, the neighborhood can be determined by comparing the received signal strength with a certain threshold (for instance $90 \mathrm{dBm}$ [28]), beyond which data packages cannot be demodulated.

- $\mathcal{H}(i)=\left\{j: j \in\left\{\mathcal{N}_{a} \cup \mathcal{N}_{b}\right\}\right.$ and $\left.d\left(\boldsymbol{p}_{i}, \boldsymbol{p}_{j}\right)<R_{c}\right\}$ is the set of all neighboring sensors of agent $i, \forall i \in \mathcal{N}_{a}$.

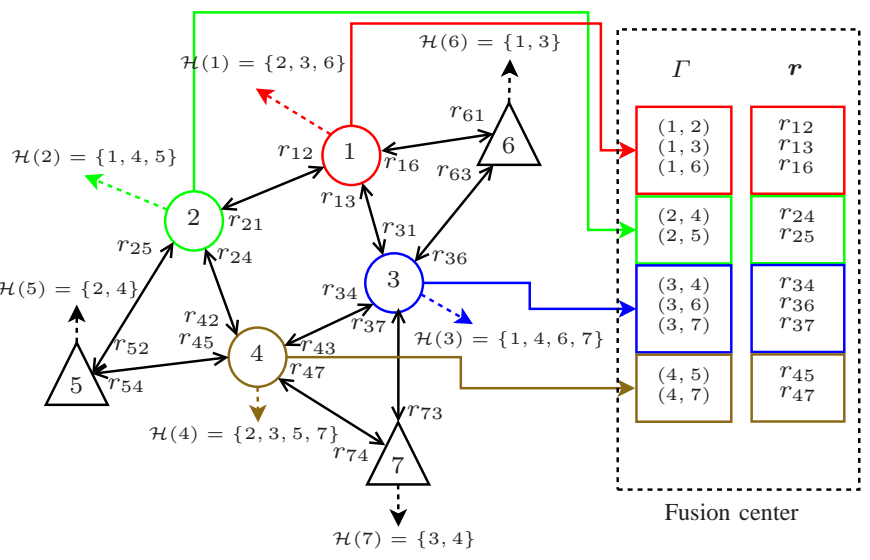

Fig. 1. An illustrating example of a connected wireless sensor network in 2-D space. In this example, there are $N=7$ sensors in total with $N_{u}=4$ agents marked by o's; the rest are anchors and marked by $\triangle$ 's. In this network, each sensor is only able to communicate with its one-hop neighbors. Each sensor collects TOA measurements with all its neighbors. All measurements that will be routed to a fusion center are stacked in $\boldsymbol{r}$, which has the same data structure as the set of feasible sensor pairs, $\Gamma$.

- $\Gamma=\left\{(i, j): i \in \mathcal{N}_{a}, j \in \mathcal{H}(i), j>i\right\}$ is a set of all sensor pairs that contribute distance measurements.

- $\boldsymbol{r}$ is a column vector of all distance measurements, $r_{i, j}$, $\forall(i, j) \in \Gamma$.

An illustrating example that better explains the above notations is given in Fig. 1.

The log-likelihood function of our joint estimation problem ${ }^{1}$ is then expressed as follows:

$$
\begin{aligned}
\mathcal{L}(\boldsymbol{\theta} ; \boldsymbol{r}) & \triangleq \ln (p(\boldsymbol{r} ; \boldsymbol{\theta}))=\sum_{i=1}^{N_{u}} \sum_{\substack{j \in \mathcal{H}(i) \\
j>i}} \ln p\left(r_{i, j} ; \boldsymbol{\theta}\right) \\
& =\sum_{(i, j) \in \Gamma} \ln \left(\sum_{l=1}^{C} \alpha_{l} \mathcal{N}\left(r_{i, j}-d\left(\boldsymbol{p}_{i}, \boldsymbol{p}_{j}\right) ; \mu_{l}, \sigma_{l}^{2}\right)\right) .
\end{aligned}
$$

The centralized MLE is obtained through solving

$$
\begin{array}{ll}
\underset{\boldsymbol{\theta}}{\operatorname{maximize}} & \mathcal{L}(\boldsymbol{\theta} ; \boldsymbol{r}) \\
\text { subject to } & 0 \leq \alpha_{1}, \ldots, \alpha_{C} \leq 1, \sum_{l=1}^{C} \alpha_{l}=1, \\
& \sigma_{l}^{2}>0, l=1,2, \ldots, C .
\end{array}
$$

This optimization problem is cumbersome for two reasons. Firstly, the cost function contains "the logarithm of the sum", which hinders the analytical evaluation of the parameters. Secondly, when the number of agents, $N_{u}$, is large, the existing numerical methods, e.g., Newton-type methods, would become less stable [29].

\section{B. Centralized ECM Algorithms}

Instead of solving (3) directly, we approximate the MLE using the ECM criterion, the idea behind which is to replace the complicated M-step of the conventional EM criterion with

\footnotetext{
${ }^{1}$ When we are deriving the new algorithms, the approximated measurement error model is assumed.
} 
a set of computationally simpler conditional maximization (CM) steps in an attempt to split a difficult maximization problem into many easier ones [29]. We first focus on the class of centralized ECM algorithms for cooperative localization, which lays a foundation for developing distributed algorithms. The centralized ECM algorithms work with a complete data set $\{\boldsymbol{r}, \boldsymbol{z}\}$, in which $\boldsymbol{z}$ is a column vector enclosing $|\Gamma|$ latent variables $z_{i, j}$ indicating which mixture component gave rise to $r_{i, j}$. Given the previous parameter estimate $\boldsymbol{\theta}^{(\eta)}$, the work-flow of the centralized ECM algorithm on the $(\eta+1)$ th iteration is as follows.

Expectation: We take conditional expectation of the complete data $\log$-likelihood $\mathcal{L}_{C}(\boldsymbol{\theta} ; \boldsymbol{r}, \boldsymbol{z}) \triangleq \ln (p(\boldsymbol{r}, \boldsymbol{z} ; \boldsymbol{\theta}))$ in terms of $\boldsymbol{z}$, given $\boldsymbol{r}$ and $\boldsymbol{\theta}^{(\eta)}$. After some tedious steps (details can be found in [30]), we obtain a $Q$-function

$$
\begin{aligned}
& Q\left(\boldsymbol{\theta} ; \boldsymbol{\theta}^{(\eta)}\right)= \\
& \sum_{(i, j) \in \Gamma} \sum_{l=1}^{C} \ln \left(\alpha_{l} \mathcal{N}\left(r_{i, j}-d\left(\boldsymbol{p}_{i}, \boldsymbol{p}_{j}\right) ; \mu_{l}, \sigma_{l}^{2}\right)\right) P_{l, i, j}^{(\eta)}
\end{aligned}
$$

with $P_{l, i, j}^{(\eta)}$ being a short-hand notation of the conditional probability $\operatorname{Pr}\left\{z_{i, j}=l \mid r_{i, j} ; \boldsymbol{\theta}^{(\eta)}\right\}$. With the aid of Bayes' rule, we have immediately

$$
P_{l, i, j}^{(\eta)} \propto \alpha_{l}^{(\eta)} \mathcal{N}\left(r_{i, j}-d\left(\boldsymbol{p}_{i}^{(\eta)}, \boldsymbol{p}_{j}^{(\eta)}\right) ; \mu_{l}^{(\eta)}, \sigma_{l}^{2,(\eta)}\right)
$$

where $\boldsymbol{p}_{j}^{(\eta)}=\boldsymbol{p}_{j}$ if sensor $j$ is an anchor.

Conditional Maximization: First, we need to find a proper partition of the unknown parameters, i.e.,

$$
\boldsymbol{\theta}=\left[\boldsymbol{\vartheta}_{1}^{T}, \boldsymbol{\vartheta}_{2}^{T}, \ldots, \boldsymbol{\vartheta}_{S}^{T}\right]^{T}
$$

where $\boldsymbol{\vartheta}_{s}$ is a sub-vector of $\boldsymbol{\theta}$. Furthermore, we let

$$
\mathcal{G}=\left\{g_{s}(\boldsymbol{\theta}): s=1,2, \ldots, S\right\}
$$

be a set of $S$ preselected vector functions of $\boldsymbol{\theta}$ defined by

$$
g_{s}(\boldsymbol{\theta}) \triangleq\left[\boldsymbol{\vartheta}_{1}^{T}, \ldots, \boldsymbol{\vartheta}_{s-1}^{T}, \boldsymbol{\vartheta}_{s+1}^{T}, \ldots, \boldsymbol{\vartheta}_{S}^{T}\right],
$$

meaning that $g_{s}(\boldsymbol{\theta})$ is a vector that contains all the sub-vectors of $\boldsymbol{\theta}$, except $\boldsymbol{\vartheta}_{s}$. With the above partition of $\boldsymbol{\theta}$, the $s$ th CM step of the $(\eta+1)$ th ECM iteration solves $\boldsymbol{\theta}^{(\eta+s / S)}$ from the following optimization problem:

$$
\begin{array}{ll}
\underset{\boldsymbol{\theta}}{\arg \max } & Q\left(\boldsymbol{\theta} ; \boldsymbol{\theta}^{(\eta)}\right) \\
\text { subject to } & g_{s}(\boldsymbol{\theta})=g_{s}\left(\boldsymbol{\theta}^{(\eta+(s-1) / S)}\right)
\end{array} .
$$

Simply speaking, when the ECM algorithm updates a subset of the parameters in a specific CM step, the rest of the parameters are fixed to their latest ECM estimate. For clarity, we give the general routine for designing a centralized ECM algorithm in Algorithm 1.

Different partitions of $\boldsymbol{\theta}$ lead to different ECM algorithms. To elaborate on this, three examples are shown below with the same partition of the mixture model parameters $\boldsymbol{\vartheta}_{1}=$ $\left[\alpha_{1}, \alpha_{2}, \ldots, \alpha_{C}\right]^{T}, \boldsymbol{\vartheta}_{2 l}=\mu_{l}, \boldsymbol{\vartheta}_{2 l+1}=\sigma_{l}^{2}, l=1,2, \ldots, C$, but with different partitions of the position parameters as follows:

- Example 1: $\boldsymbol{\vartheta}_{2 C+1+i}=\boldsymbol{p}_{i}, i=1,2, \ldots, N_{u}$, hence $S=$ $N_{u}+2 C+1$.

\footnotetext{
$\overline{\text { Algorithm } 1 \text { Centralized ECM Algorithm for Cooperative }}$ Localization (General Routine)

Initialization: Set a convergence tolerance $\Delta_{c}$ and the maximum number of iterations to $N_{i t r}^{E}$; Choose an initial guess $\boldsymbol{\theta}^{(\eta=0)}$.

Expectation-Conditional Maximization:

On the $(\eta+1)$ th iteration $(\eta \in \mathbb{Z}, \eta \geq 0)$, do:

1) Perform conditional expectation and obtain $Q\left(\boldsymbol{\theta} ; \boldsymbol{\theta}^{(\eta)}\right)$, cf.(4).

2) Find a proper partition of $\boldsymbol{\theta}=\left[\boldsymbol{\vartheta}_{1}^{T}, \ldots, \boldsymbol{\vartheta}_{S}^{T}\right]^{T}$. See for instance the three examples given below.

3) Find $\boldsymbol{\theta}^{(\eta+s / S)}$ that solves (5) for $s=1,2, \ldots, S$.

\section{Convergence Check:}

If $\mathcal{L}\left(\boldsymbol{\theta}^{(\eta+1)} ; \boldsymbol{r}\right)-\mathcal{L}\left(\boldsymbol{\theta}^{(\eta)} ; \boldsymbol{r}\right) \leq \Delta_{c}$ or the maximum number of iterations $N_{i t r}^{E}$ has been reached, then stop; otherwise reset $\eta \leftarrow \eta+1$ and return to the ECM stage.
}

- Example 2: $\boldsymbol{\vartheta}_{2 C+2 i}=x_{i}$ and $\boldsymbol{\vartheta}_{2 C+2 i+1}=y_{i}, i=$ $1,2, \ldots, N_{u}$, hence $S=2 N_{u}+2 C+1$.

- Example 3: $\boldsymbol{\vartheta}_{2 C+2}=\boldsymbol{\theta}_{p}=\left[\boldsymbol{p}_{1}^{T}, \ldots, \boldsymbol{p}_{N_{u}}^{T}\right]^{T}$, hence $S=$ $2 C+2$.

Applying the above partition of the Gaussian mixture model parameters and following the steps shown in [25] to update $\alpha_{l}^{(\eta+1)}, \mu_{l}^{(\eta+1)}, \sigma_{l}^{2,(\eta+1)}$, for every $l=1,2, \ldots, C$, yields

$$
\begin{gathered}
\alpha_{l}^{(\eta+1)}=\frac{1}{|\Gamma|} \sum_{(i, j) \in \Gamma} P_{l, i, j}^{(\eta)}, \\
\mu_{l}^{(\eta+1)}=\frac{\sum_{(i, j) \in \Gamma}\left(r_{i, j}-d\left(\boldsymbol{p}_{i}^{(\eta)}, \boldsymbol{p}_{j}^{(\eta)}\right)\right) P_{l, i, j}^{(\eta)}}{|\Gamma| \alpha_{l}^{(\eta+1)}}, \\
\sigma_{l}^{2,(\eta+1)}=\frac{\sum_{(i, j) \in \Gamma}\left(r_{i, j}-d\left(\boldsymbol{p}_{i}^{(\eta)}, \boldsymbol{p}_{j}^{(\eta)}\right)\right)^{2} P_{l, i, j}^{(\eta)}}{|\Gamma| \alpha_{l}^{(\eta+1)}}-\left(\mu_{l}^{(\eta+1)}\right)^{2} .
\end{gathered}
$$

It is easy to verify that for any $s=1,2, \ldots, 2 C+1$,

$$
Q\left(\boldsymbol{\theta}^{(\eta+s / S)} ; \boldsymbol{\theta}^{(\eta)}\right) \geq Q\left(\boldsymbol{\theta} ; \boldsymbol{\theta}^{(\eta)}\right)
$$

holds for any $\boldsymbol{\theta} \in \Theta_{s}\left(\boldsymbol{\theta}^{(\eta+(s-1) / S)}\right) \triangleq\left\{\boldsymbol{\theta} \in \Theta: g_{s}(\boldsymbol{\theta})=\right.$ $\left.g_{s}\left(\boldsymbol{\theta}^{(\eta+(s-1) / S)}\right)\right\}$. In other words, $\boldsymbol{\theta}^{(\eta+s / S)}$ is the global maximizer in the given subspace of $\Theta$.

The position updates can be evaluated only numerically. For the first example, it can be shown that

$$
\boldsymbol{p}_{i}^{(\eta+1)}=\underset{\boldsymbol{p}_{i}}{\arg \min } \sum_{j \in \mathcal{H}(i)} \sum_{l=1}^{C} \frac{\left(r_{i, j}-d\left(\boldsymbol{p}_{i}, \boldsymbol{p}_{j}^{(\tilde{\eta})}\right)-\mu_{l}^{(\eta+1)}\right)^{2}}{\sigma_{l}^{2,(\eta+1)} / P_{l, i, j}^{(\eta)}}
$$

for $i=1,2, \ldots, N_{u}$. The neat formulation of the cost function in (9) is due to the assumption $r_{i, j}=r_{j, i}$. In (9), $\boldsymbol{p}_{j}^{(\tilde{\eta})}=\boldsymbol{p}_{j}^{(\eta+1)}$ if sensor $j$ is an agent with its position updated prior to sensor $i$, or $\boldsymbol{p}_{j}^{(\tilde{\eta})}=\boldsymbol{p}_{j}^{(\eta)}$ otherwise; or $\boldsymbol{p}_{j}^{(\tilde{\eta})}=\boldsymbol{p}_{j}$ if sensor $j$ is an anchor. Herein, we adopt a two-dimensional (2D) Broyden-Fletcher-Goldfarb-Shanno (BFGS) quasi-Newton (QN) method [31] to solve $\boldsymbol{p}_{i}^{(\eta+1)}$, because it ensures

$$
Q\left(\boldsymbol{\theta}^{(\eta+(2 C+1+i) / S)} ; \boldsymbol{\theta}^{(\eta)}\right) \geq Q\left(\boldsymbol{\theta}^{(\eta+(2 C+i) / S)} ; \boldsymbol{\theta}^{(\eta)}\right),
$$

for $i=1,2, \ldots, N_{u}$. We stress that the positions need not to be updated in any specified order of the sensor indices. 
In the second example, the position update $\left[x_{i}^{(\eta+1)}, y_{i}^{(\eta+1)}\right]$ of each agent is found by applying one-dimensional (1-D) grid search (GS), respectively, to

$$
\begin{aligned}
& x_{i}^{(\eta+1)}= \\
& \underset{x_{i}}{\arg \min } \sum_{j \in \mathcal{H}(i)} \sum_{l=1}^{C} \frac{\left(r_{i, j}-d\left(\left[x_{i}, y_{i}^{(\eta)}\right]^{T}, \boldsymbol{p}_{j}^{(\tilde{\eta})}\right)-\mu_{l}^{(\eta+1)}\right)^{2}}{\sigma_{l}^{2,(\eta+1)} / P_{l, i, j}^{(\eta)}},
\end{aligned}
$$

$$
\begin{gathered}
y_{i}^{(\eta+1)}= \\
\underset{y_{i}}{\arg \min } \sum_{j \in \mathcal{H}(i)} \sum_{l=1}^{C} \frac{\left(r_{i, j}-d\left(\left[x_{i}^{(\eta+1)}, y_{i}\right]^{T}, \boldsymbol{p}_{j}^{(\tilde{\eta})}\right)-\mu_{l}^{(\eta+1)}\right)^{2}}{\sigma_{l}^{2,(\eta+1)} / P_{l, i, j}^{(\eta)}} .
\end{gathered}
$$

With carefully selected search intervals together with a fine grid resolution, global maximizer of (5) is reachable in the given subspace for each CM step. As a trade-off, in total $2 N_{u}$ trials 1-D grid searches need to be performed for each iteration, which consumes more computational resources than the 2-D BFGS-QN based ECM algorithm derived for the first example.

Essentially, the third example corresponds to our previous work in [25], which can also be regarded as an ECM algorithm with the partition given beforehand. We note that [25] is more suitable to use for small or moderate $N_{u}$, because intuitively it should converge much faster. A good example was given in [32], where only one $\left(N_{u}=1\right)$ mobile station is to be located in a non-cooperative framework. The first and second ECM algorithms are, however, favorable for a large $N_{u}$, since they should be numerically more stable [29]. In the remainder of this paper, we only focus on the first two examples because they allow for easier decentralization as will be seen in Section III-C.

Remark 1: For the special case when $C=1$ in (2), the Gaussian mixture model degenerates to the single Gaussian model. It is easy to verify that in this case $\mathcal{L}(\boldsymbol{\theta} ; \boldsymbol{r})=$ $Q\left(\boldsymbol{\theta} ; \boldsymbol{\theta}^{(\eta)}\right)$ holds with $C=1, \alpha_{1}=1$, and $P_{1, i, j}^{(\eta)}=1$ in (4). It is also easy to show that the parameter estimates can be updated by setting $l=C=1, P_{1, i, j}^{(\eta)}=1$, and $\alpha_{1}^{(\eta+1)}=1$ in (7), (8), and (9) for the 2-D BFGS-QN based algorithm, or in (10) and (11) for the 1-D GS based algorithm. In contrast to the other cases where $C>1$, only an initial guess of the agent positions is required for the ECM algorithms to start computing $\mu_{l}^{(1)}$ and $\sigma_{1}^{2,(0)}$ simply because $P_{1, i, j}^{(\eta)}$ is a constant. ${ }^{2}$

Remark 2: The centralized ECM algorithms enumerated in the examples above ensure that

$$
\begin{aligned}
Q\left(\boldsymbol{\theta}^{(\eta+1)} ; \boldsymbol{\theta}^{(\eta)}\right) & \geq Q\left(\boldsymbol{\theta}^{(\eta+(S-1) / S)} ; \boldsymbol{\theta}^{(\eta)}\right) \\
& \geq Q\left(\boldsymbol{\theta}^{(\eta+(S-2) / S)} ; \boldsymbol{\theta}^{(\eta)}\right) \\
& \vdots \\
& \geq Q\left(\boldsymbol{\theta}^{(\eta)} ; \boldsymbol{\theta}^{(\eta)}\right),
\end{aligned}
$$

\footnotetext{
${ }^{2}$ When deriving $\mu_{l}^{(\eta+1)}$ from $(5), \sigma_{1}^{2,(\eta)}$ can in fact take any real value that is larger than zero, since it will be canceled out during the calculation.
}

which is equivalently to saying that $\mathcal{L}\left(\boldsymbol{\theta}^{(\eta+1)} ; \boldsymbol{r}\right) \geq$ $\mathcal{L}\left(\boldsymbol{\theta}^{(\eta)} ; \boldsymbol{r}\right)$. Hence, they also belong to the class of generalized EM (GEM) algorithms [33].

Theorem 1: When the position updates found either by 2D BFGS-QN search (9) or 1-D grid search (10) and (11) are global maximizers, the proposed centralized ECM algorithms ensure that the sequence of incomplete-data log-likelihood values $\left\{\mathcal{L}\left(\boldsymbol{\theta}^{(\eta)} ; \boldsymbol{r}\right)\right\}$, when bounded above, converges monotonically to some stationary point $L^{*}$, satisfying that $L^{*}=$ $\mathcal{L}\left(\boldsymbol{\theta}^{*} ; \boldsymbol{r}\right)$ and $\partial \mathcal{L}(\boldsymbol{\theta} ; \boldsymbol{r}) / \partial \boldsymbol{\theta}=\mathbf{0}$ at $\boldsymbol{\theta}^{*}{ }^{3}$

Proof: See Appendix A.

Remark 3: The proposed centralized ECM algorithms may converge to different stationary points if $\mathcal{L}(\boldsymbol{\theta} ; \boldsymbol{r})$ is multimodal. The reason is that different partitions of $\boldsymbol{\theta}$ lead to different mappings $\boldsymbol{\theta}^{(\eta+1)}=M\left(\boldsymbol{\theta}^{(\eta)}\right)$ in the conditional maximization. For the special case that $\mathcal{L}(\boldsymbol{\theta} ; \boldsymbol{r})$ is unimodal, they will reach a unique global maximum.

\section{Distributed ECM Algorithms}

Distributed self-localization algorithms are scalable in general and have the potential to largely reduce the communication overhead, making them highly demanded for largescale sensor networks [20]. The aim of this subsection is to approximate the centralized ECM algorithms in a distributed manner. Essentially, the above centralized ECM algorithms can be understood as an iterative process consisting of the following two steps on each iteration: (1) updating the mixture model parameters $\boldsymbol{\theta}_{m}$ with the position parameters $\boldsymbol{\theta}_{p}$ held fixed to its previous estimate; and (2) updating the position parameters $\boldsymbol{\theta}_{p}$ with the Gaussian mixture model parameters $\boldsymbol{\theta}_{m}$ held fixed to its latest estimate. We decentralize these two steps in the sequel.

We start with decentralizing the first step. The results in (6)-(8) can be re-expressed, after some simple manipulations, as:

$$
\begin{gathered}
\alpha_{l}^{(\eta+1)}=\frac{N_{u}}{|\Gamma|} \frac{1}{N_{u}} \sum_{i=1}^{N_{u}} w_{i, l} \propto \frac{1}{N_{u}} \sum_{i=1}^{N_{u}} w_{i, l}, \\
\mu_{l}^{(\eta+1)}=\frac{\frac{1}{N_{u}} \sum_{i=1}^{N_{u}} a_{i, l}}{\frac{1}{N_{u}} \sum_{i=1}^{N_{u}} w_{i, l}}
\end{gathered}
$$

$$
\sigma_{l}^{2,(\eta+1)}=\frac{\frac{1}{N_{u}} \sum_{i=1}^{N_{u}} b_{i, l}}{\frac{1}{N_{u}} \sum_{i=1}^{N_{u}} w_{i, l}}-\left(\mu_{l}^{(\eta+1)}\right)^{2},
$$

\footnotetext{
${ }^{3}$ In this paper, a stationary point can be a saddle point, a local optimum or a global optimum.
} 
where

$$
\begin{aligned}
w_{i, l} & =\sum_{\substack{j \in \mathcal{H}(i), j>i}} P_{l, i, j}^{(\eta)}, \\
a_{i, l} & =\sum_{\substack{j \in \mathcal{H}(i), j>i}} P_{l, i, j}^{(\eta)}\left(r_{i, j}-d\left(\boldsymbol{p}_{i}^{(\eta)}, \boldsymbol{p}_{j}^{(\eta)}\right)\right), \\
b_{i, l} & =\sum_{\substack{j \in \mathcal{H}(i), j>i}} P_{l, i, j}^{(\eta)}\left(r_{i, j}-d\left(\boldsymbol{p}_{i}^{(\eta)}, \boldsymbol{p}_{j}^{(\eta)}\right)\right)^{2} .
\end{aligned}
$$

It is clear from (13)-(15) that averaging is the main operation in common for updating the Gaussian mixture model parameters. This allows us to use the class of average consensus algorithms [34], because it does not rely on a prescribed route, like Hamiltonian path in [35], [36] or some tree structure in [14], and thus is more robust against sensor malfunction and link failure. Here, we assume for simplicity that the sensors are time synchronized and adapt the synchronous average consensus algorithm [37] to our problem in Algorithm 2. Our motivations are in order. On the one hand, it is easier to implement and an efficient protocol [38] exists. On the other hand, its synchronous nature facilitates our subsequent analyses on the computational complexity and communication overhead. However, we note that Algorithm 2 can be reformulated asynchronously (known as the "pair-wise gossip algorithm" [39]) or replaced with other consensus based methods [40] or diffusion based methods [41]. Lastly, we note that in the distributed algorithms $P_{l, i, j}^{(\eta)}$ in (16)-(18) has to be replaced by

$$
\tilde{P}_{l, i, j}^{(\eta)} \propto \alpha_{i, l}^{(\eta)} \mathcal{N}\left(r_{i, j}-d\left(\boldsymbol{p}_{i}^{(\eta)}, \boldsymbol{p}_{j}^{(\eta)}\right) ; \mu_{i, l}^{(\eta)}, \sigma_{i, l}^{2,(\eta)}\right)
$$

which can be computed locally at each agent $i$.

$\overline{\text { Algorithm } 2 \text { Synchronous Average Consensus Algorithm (for }}$ Agent $i$ on the $(\eta+1)$ th ECM Iteration)

1. Repeat 1.a. and 1.b. for $N_{i t r}^{G}$ iterations:

1.a. Choose a neighboring agent $j$ uniformly at random from $\mathcal{H}(i)$. Send $\left\{w_{i, l}^{(c)}, a_{i, l}^{(c)}, b_{i, l}^{(c)}\right\}$ to and receive $\left\{w_{j, l}^{(c)}, a_{j, l}^{(c)}, b_{j, l}^{(c)}\right\}$ from agent $j$.

1.b. Agents $i$ and $j$ update

$$
\chi_{i, l}^{(n)}=\chi_{j, l}^{(n)}=\frac{\chi_{i, l}^{(c)}+\chi_{j, l}^{(c)}}{2}
$$

and re-set $\chi_{i, l}^{(c)}=\chi_{i, l}^{(n)}$, where $\chi \in\{w, a, b\}$.

Note that $\left\{w_{i, l}^{(c)}, a_{i, l}^{(c)}, b_{i, l}^{(c)}\right\}$ is initialized by $\left\{w_{i, l}, a_{i, l}, b_{i, l}\right\}$ computed by (16)-(18) but with $P_{l, i, j}^{(\eta)}$ replaced by $\tilde{P}_{l, i, j}^{(\eta)}$ therein.

2. Update the local estimate of the Gaussian mixture model parameters by

$$
\alpha_{i, l}^{(\eta+1)} \propto w_{i, l}^{(c)}, \mu_{i, l}^{(\eta+1)}=\frac{a_{i, l}^{(c)}}{w_{i, l}^{(c)}}, \sigma_{i, l}^{2,(\eta+1)}=\frac{b_{i, l}^{(c)}}{w_{i, l}^{(c)}}-\left(\mu_{i, l}^{(\eta+1)}\right)^{2} .
$$

Note that $\alpha_{i, l}^{(\eta+1)}, l=1,2, \ldots, C$ need to be scaled to sum up to one.

The position update procedures in the centralized ECM algorithms are readily in decentralized form because the evaluation of (9) or (10)-(11) only requires that each agent solves for its own position, given the local measurements, the updated Gaussian mixture model parameters, and the neighboring sensor positions. Only two modifications are needed. First, the global estimates $\mu_{l}^{(\eta+1)}$ and $\sigma_{l}^{2,(\eta+1)}$ in (9) or (10)(11) have to be replaced with the local estimates $\mu_{i, l}^{(\eta+1)}$ and $\sigma_{i, l}^{2,(\eta+1)}$ for each $i$. Second, a synchronous position update scheme, in which all agents are able to update their positions simultaneously, requires $\boldsymbol{p}_{j}^{(\tilde{\eta})}$ to be substituted with $\boldsymbol{p}_{j}^{(\eta)}$. Algorithm 3 summarizes the key steps of the two distributed ECM algorithms.

$\overline{\text { Algorithm } 3 \text { Distributed ECM Algorithms (for Agent } i \text { on the }}$ $(\eta+1)$ th ECM Iteration)

Initialization: Find initial guesses $\boldsymbol{\theta}_{m}^{(0)}, \boldsymbol{p}_{i}^{(0)}$, and $\boldsymbol{p}_{j}^{(0)}, \forall j \in \mathcal{H}(i)$. Set the maximum number of iterations $N_{i t r}^{E}$.

Expectation-Conditional Maximization:

On the $(\eta+1)$ th iteration $(\eta \in \mathbb{Z}, \eta \geq 0)$, agent $i$ does:

1) Compute $\tilde{P}_{l, i, j}^{(\eta)}, \forall l=1,2, \ldots, C$ and $\forall j \in \mathcal{H}(i)$ according to (19), and $\chi_{i, l}^{(c)}, \forall l=1,2, \ldots, C$ and $\forall \chi \in\{w, a, b\}$ according to (16)-(18) with $P_{l, i, j}^{(\eta)}$ replaced by $\tilde{P}_{l, i, j}^{(\eta)}$ therein.

2) Run Algorithm 2 to compute $\alpha_{i, l}^{(\eta+1)}, \mu_{i, l}^{(\eta+1)}$, and $\sigma_{i, l}^{2,(\eta+1)}$, $l=1,2, \ldots, C$

3) Solve $\boldsymbol{p}_{i}^{(\eta+1)}=\left[x_{i}^{(\eta+1)}, y_{i}^{(\eta+1)}\right]^{T}$ using

- either the 2-D BFGS-QN method according to (9);

- or the 1-D GS method according to (10) and (11).

Note that we need to replace $P_{l, i, j}^{(\eta)}$ by $\tilde{P}_{l, i, j}^{(\eta)}, \mu_{l}^{(\eta+1)}$ by $\mu_{i, l}^{(\eta+1)}$, $\sigma_{l}^{2,(\eta+1)}$ by $\sigma_{i, l}^{2,(\eta+1)}$, and $\boldsymbol{p}_{j}^{(\tilde{\eta})}$ by $\boldsymbol{p}_{j}^{(\eta)}$ in (9) or in (10) and (11).

4) Broadcast $\boldsymbol{p}_{i}^{(\eta+1)}$ to its neighbors.

\section{Convergence Check:}

If $N_{i t r}^{E}$ has been reached, then terminate; otherwise reset $\eta \leftarrow \eta+1$ and return to the ECM stage.

The position estimate $\boldsymbol{p}_{i}^{(\eta+1)}$ in the 2-D BFGS-QN based distributed ECM algorithm can be imagined as a weighted least-squares (WLS) solution for a conventional infrastructure based localization problem, in which a total number of $|\mathcal{H}(i)|$ virtual anchors with positions $\boldsymbol{p}_{j}^{(\eta)}, j \in \mathcal{H}(i)$ surround agent $i$ and each virtual anchor (say the $j$ th) has a number of $C$ distance measurements $r_{i, j}-\mu_{i, l}^{(\eta+1)}$ corrupted by errors with zero mean and variances $\sigma_{i, l}^{2,(\eta+1)} / \tilde{P}_{l, i, j}^{(\eta)}$. For problems of this kind, many existing linearization strategies, see for instance [42, Chapter 2], can be used to further reduce the computational complexity.

\section{Initialization Strategy}

We provide a practical solution for initializing the distributed ECM algorithms. First, an initial guess of the Gaussian mixture model parameters is determined by carrying out the conventional Gaussian mixture learning between two communicating anchors. This can be done prior to collecting distance measurements among all sensors. Alternatively, we could also empirically choose one in order to save some communication cost. However, this might incur performance degradation. An initial guess of the unknown agent positions is determined in a simple algorithm, for instance, the distributed least-squares algorithm [5, Algorithm 1] with a starting point randomly selected from the deployed area. An initial guess 
for the centralized ECM algorithms can be determined more easily, since the fusion center usually has all the necessary information (e.g., measurements and prior knowledge) and sufficient computation resources.

\section{E. Possible Extension}

In Section III-B, the centralized ECM algorithms were developed based on the assumption that $r_{i, j}=r_{j, i}$ so as to reduce c.a. half of the communication energy for multihop routing of data. If this assumption does not hold, all the measurements should be used to give better estimation performance. In order to adapt the above introduced ECM algorithms, we need to re-define $\Gamma=\left\{(i, j): i \in \mathcal{N}_{a} \cup \mathcal{N}_{b}, j \in\right.$ $\mathcal{H}(i)\}$ and re-express the $Q$-function in (4) as

$$
\begin{aligned}
& Q\left(\boldsymbol{\theta} ; \boldsymbol{\theta}^{(\eta)}\right)= \\
& \quad \sum_{i=1}^{N} \sum_{j \in \mathcal{H}(i)} \sum_{l=1}^{C} \ln \left(\alpha_{l} \mathcal{N}\left(r_{i, j}-d\left(\boldsymbol{p}_{i}, \boldsymbol{p}_{j}\right) ; \mu_{l}, \sigma_{l}^{2}\right)\right) P_{l, i, j}^{(\eta)} .
\end{aligned}
$$

The centralized (global) parameter estimates can be found using the same strategy shown in Section III-B and the explicit expressions are given in Appendix B. The distributed ECM algorithms aim to approximate the performance of their centralized counterparts and can be obtained using the same technique demonstrated in Section III-C except that: (1) all the sensors (both the agents and anchors) will be involved in the average consensus algorithm; (2) when agent $i$ updates its own position, both $\left\{r_{i, j}, P_{l, i, j}^{(\eta)}\right\}$ and $\left\{r_{j, i}, P_{l, j, i}^{(\eta)}\right\}$ for all $j \in \mathcal{H}(i)$ are required. Clearly, this will introduce extra cost for wireless communication; and (3) the computational complexity is also doubled.

Lastly, we want to mention that in the centralized ECM algorithms (see Section III-B) some sensor nodes would transmit more data packages to the fusion center than the others, and this would lead to an uneven communication load. Actually, this problem yet exists in the modified centralized ECM algorithms (see Section III-E), because in general different sensors have different number of neighbors, $|\mathcal{H}(i)|$, thus different number of measurements to transmit. Nevertheless, the distributed ECM algorithms are totally free of uneven communication load, irrespective of the assumption $\left(r_{i, j}=r_{j, i}\right.$ or $r_{i, j} \neq r_{j, i}$ ) based on which they have been developed. In the simulations, we only test the centralized ECM algorithms proposed in Section III-B and the distributed ECM algorithms in Section III-C.

\section{COMPUtational COMPlexity AND COMMUNiCATION OVERHEAD}

Computational complexity, communication overhead (or energy consumption for communication), and localization accuracy are key aspects of a cooperative localization algorithm. This section covers the first two aspects but lays more emphasis on the distributed algorithms.

\section{A. Computational Complexity}

For simplicity the complexity analysis is conducted for a single agent (say the $i$ th) to run one single iteration. We start with the 2-D BFGS-QN based distributed ECM algorithm, cf. Algorithm 3, and focus on the expectation-conditional maximization stage. In the first step, the computation of $\tilde{P}_{l, i, j}^{(\eta)}$, $\forall j \in \mathcal{H}(i)$ according to (19) and $\chi_{i l}^{(c)}, \forall \chi \in\{w, a, b\}$ according to (16)-(18) for all $l=1,2, \ldots, C$ is in the order $\mathcal{O}(C|\mathcal{H}(i)|)$. In the second step, local estimates of the Gaussian mixture model parameters are computed in an average consensus algorithm, cf. Algorithm 2, whose complexity scales as $\mathcal{O}\left(N_{i t r}^{G} C\right)$. In the last step, position update $p_{i}^{(\eta+1)}$ is found numerically via a Newton-type method (iterative in nature). Similar to [32], it can be shown that the complexity scales as $\mathcal{O}\left(N_{i t r}^{n u} C|\mathcal{H}(i)|\right)$, where $N_{i t r}^{n u}$ is defined to be the total number of Newton iterations. Therefore, the complexity for the $i$ th agent to run one iteration is $\mathcal{O}\left(C|\mathcal{H}(i)|\left(N_{i t r}^{n u}+N_{i t r}^{G} /|\mathcal{H}(i)|\right)\right)$, which can be approximated by $\mathcal{O}\left(C|\mathcal{H}(i)| N_{i t r}^{n u}\right)$ when $N_{i t r}^{G}$ is small. The complexity of the corresponding centralized ECM algorithm is easy to obtain as $\mathcal{O}\left(N_{u} N_{i t r}^{n u} C\left|\mathcal{H}_{\text {ave }}\right|\right)$, where $\left|\mathcal{H}_{\text {ave }}\right| \triangleq 1 / N_{u} \sum_{i=1}^{N_{u}}|\mathcal{H}(i)|$ is the average number of neighbors computed among all agents. Assuming that the sensors are uniformly distributed in a 2 -D area with $|\mathcal{H}(i)| \approx\left|\mathcal{H}_{\text {ave }}\right|, \forall i=1,2, \ldots, N_{u}$, the computational power required by the centralized ECM algorithm is nearly evenly distributed to every agent. Similar conclusion can be drawn for the 1-D GS based algorithms, as it is easy to verify that the complexity of the distributed algorithm scales as $\mathcal{O}\left(C|\mathcal{H}(i)| R_{\text {grid }}\right)$ and the corresponding centralized algorithm $\mathcal{O}\left(N_{u} R_{\text {grid }} C\left|\mathcal{H}_{\text {ave }}\right|\right)$, where $R_{\text {grid }}$ is defined to be the number of grid points used in the 1-D search of $x_{i}$ or $y_{i}$.

Next, we compare the complexity of various different distributed algorithms. The results are given in Table I. Therein, for brevity, "2-D BFGS-QN D(C)-ECM" denotes the distributed (centralized) ECM algorithm using 2-D BFGS-QN search of the positions and "1-D GS D(C)-ECM" denotes the distributed (centralized) ECM algorithm using 1-D GS of the positions. It is noteworthy that $R_{\text {int }}$ denotes the number of particles used to represent the internal messages (distributions) for both the sample-based and parametric SPAWN algorithms. With the help of the typical value of the design parameters given in Table I, we can conclude the following. Firstly, the distributed least-squares (LS) algorithm and the distributed weighted MDS (dwMDS) algorithm have the lowest computational complexity, as they do not require iterations within an iteration as compared to the distributed ECM algorithms or particle representation of the messages as compared to the SPAWN algorithms. Secondly, the complexity of the 2D BFGS-QN D-ECM algorithm is expected to be lower than that of the SPAWN algorithms. This is because $N_{i t r}^{n u} C$ should be, in general, smaller than $R_{\text {int }}$ and negligible as compared to $R_{\text {int }}^{2}$. While the complexity of the 1-D GS D-ECM algorithm should be comparable with that of the parametric SPAWN algorithms but much lower than that of the conventional SPAWN algorithm.

\section{B. Communication Overhead}

Generally speaking, a centralized, cooperative localization algorithm spends most of the energy on the wireless routing of 
TABLE I

Computational Complexity for An Agent (SAY the $i$ Th) To Run ONE ITERATION OF DIFFERENT DISTRIBUTED ALGORITHMS

\begin{tabular}{|l|l|l|}
\hline Distributed Algorithm & Complexity & Typical Value \\
\hline Least-Squares [5] & $\mathcal{O}(|\mathcal{H}(i)|)$ & \\
\hline Weighted MDS [8] & $\mathcal{O}(|\mathcal{H}(i)|)$ & \\
\hline Parametric SPAWN [15], [16] & $\mathcal{O}\left(R_{\text {int }}|\mathcal{H}(i)|\right)$ & $R_{\text {int }}: 10^{2}-10^{3}$ \\
\hline Classical SPAWN [5] & $\mathcal{O}\left(R_{\text {int }}^{2}|\mathcal{H}(i)|\right)$ & $R_{\text {int }}^{2}: 10^{4}-10^{6}$ \\
\hline 2-D BFGS-QN D-ECM & $\mathcal{O}\left(N_{\text {itr }}^{\text {nu }} C|\mathcal{H}(i)|\right)$ & $\begin{array}{l}C: 10^{0}-10^{1} \\
N_{i t r}^{n u}: 10^{1}-10^{2}\end{array}$ \\
\hline 1-D GS D-ECM & $\mathcal{O}\left(R_{\text {grid }} C|\mathcal{H}(i)|\right)$ & $\begin{array}{l}C: 10^{0}-10^{1} \\
R_{\text {grid }}: 10^{2}-10^{3}\end{array}$ \\
\hline
\end{tabular}

the collected measurements via multi-hops to a fusion center. While a distributed self-localization algorithm spends most of the energy on the wireless transmission of refined point estimates (for non-Bayesian algorithms) or particles (for Bayesian algorithms) among neighboring sensor pairs. This is due to the fact that the energy consumed for transmitting one bit far outweighs that consumed for executing a single instruction on board [43]. We follow the methodology proposed in [36], [44] to quantitatively analyze the total energy consumption. Some assumptions are made as follows:

(A1) All sensors are uniformly distributed over a 2-D unit square.

(A2) A multi-hop communication model is considered, and the threshold of one-hop distance is $R_{c}=\mathcal{O}\left(N_{u}^{-1 / 2}\right)$.

(A3) A real value is represented in double-precision floatingpoint format (64-bit precision).

The total energy consumed for communication by any cooperative localization algorithm can be written as

$$
\mathcal{E}\left(N_{u}\right)=b\left(N_{u}\right) \times h\left(N_{u}\right) \times e\left(N_{u}\right)
$$

where $b\left(N_{u}\right)$ is the total number of transmitted bits, $h\left(N_{u}\right)$ is the average number of hops required for transmitting one bit to the destination, and $e\left(N_{u}\right)$ is the average amount of energy required for transmitting one bit over one hop.

In the sequel, we first calculate the total energy consumed by all agents to run one single ECM iteration. In the centralized ECM algorithms, the number of real values to be transmitted to the fusion center is

$$
|\Gamma| \approx \sum_{i=1}^{N_{u}} \frac{|\mathcal{H}(i)|}{2}=N_{u}\left|\mathcal{H}_{\text {ave }}\right| .
$$

The approximation in (20) is due to the assumptions that only one of $r_{i, j}$ and $r_{j, i}$ is used and $N_{u} \approx N$. Since a real value is represented in 64-bit precision, we have

$$
b_{\text {cen }}\left(N_{u}\right)=\mathcal{O}\left(N_{u}\left|\mathcal{H}_{\text {ave }}\right|\right) \text { bits. }
$$

The average number of hops from an agent to a fusion center is $h_{\text {cen }}\left(N_{u}\right)=\mathcal{O}\left(N_{u}^{1 / 2}\right)$ according to the first assumption. The total energy consumption for the centralized ECM algorithms is therefore

$$
\mathcal{E}_{\text {cen }}\left(N_{u}\right)=\mathcal{O}\left(\left|\mathcal{H}_{\text {ave }}\right| N_{u}^{3 / 2}\right) \times e\left(N_{u}\right)
$$

In the two distributed ECM algorithms given in Algorithm 3, the total number of real values exchanged among neighboring sensors on one iteration is approximately equal
TABLE II

ENERGY CONSUMED By ALL SENSORS TO RUN ONE ITERATION OF DIFFERENT DISTRIBUTED ALGORITHMS

\begin{tabular}{|l|l|l|}
\hline Distributed Algorithm & Energy Cost & Typical Value \\
\hline Least-Squares [5] & $\mathcal{O}\left(N_{u}\right)$ & \\
\hline Weighted MDS [8] & $\mathcal{O}\left(N_{u}\right)$ & \\
\hline Parametric SPAWN [15], [16] & $\mathcal{O}\left(C_{\text {bel }} N_{u}\right)$ & $C_{\text {bel }}: 10^{0}-10^{1}$ \\
\hline Classical SPAWN [5] & $\mathcal{O}\left(R_{b e l} N_{u}\right)$ & $R_{\text {bel }}: 10^{2}-10^{3}$ \\
\hline D-ECMs & $\mathcal{O}\left(C N_{i t r}^{G} N_{u}\right)$ & $\begin{array}{l}C: 10^{0}-10^{1} \\
N_{i t r}^{G}: 10^{0}-10^{1}\end{array}$ \\
\hline
\end{tabular}

to $6 N_{u} N_{i t r}^{G} C$. Note that much less energy is consumed for updating the position estimate than for updating the mixture model parameter estimate via Algorithm 2. Therefore,

$$
b_{\text {dis }}\left(N_{u}\right)=\mathcal{O}\left(N_{u} N_{i t r}^{G} C\right) \text { bits. }
$$

Since all the data are communicated between one-hop neighboring sensors, we have $h_{d i s}\left(N_{u}\right)=1$. Therefore, the energy consumed by the distributed ECM algorithms for one iteration is given by

$$
\mathcal{E}_{\text {dis }}\left(N_{u}\right)=\mathcal{O}\left(N_{u} N_{i t r}^{G} C\right) \times e\left(N_{u}\right) .
$$

For comparison purposes, the energy consumption of different distributed algorithms are listed in Table II, where the common factor $e\left(N_{u}\right)$ has been omitted. The parameter $R_{b e l}$ in the classical SPAWN algorithm [5] denotes the number of particles required to represent each local belief message. In general, $R_{\text {bel }}$ is large. In order to reduce the energy consumption, some new fashioned SPAWN algorithms, e.g., [15] and [16], resort to fit the local belief messages with parametric models and exchange merely a few parametric model parameters between sensors. Here, $C_{b e l}$ denotes the total number of parameters used in a parametric model, which should be much less than $R_{\text {bel }}$. Gathering the above analysis and the results shown in Table II, it is easy to conclude the following. When the number of agents, $N_{u}$ tends to infinity, while the other design parameters are held constant, all the distributed algorithms scale as $\mathcal{O}\left(N_{u}\right)$ in the end and the centralized ECM algorithms consume the largest amount of energy. But for a fixed $N_{u}$, the scale of the other design parameters would also influence the actual communication cost. Given the typical values in Table II, we can pin down that: 1) the distributed LS algorithm and the dwMDS algorithm consume the smallest amount of energy for one single iteration; 2) the energy consumed by the distributed ECM algorithms should be comparable with that of the parametric SPAWN variations whereas less than that of the classical SPAWN.

\section{Concluding Remark}

In the previous subsections, all the selected distributed algorithms are iterative in nature. For clarity, the analyses were carried out only for one single iteration. However, it is noteworthy that the total computational time and communication overhead required to fully run a distributed algorithm also rely on the total number of iterations, $N_{i t r}$, which will be further elaborated in Section VI. 


\section{FUndAMENTAL Limits OF LOCALIZATION ACCURACY}

This section aims to derive the best achievable localization accuracy as a function of the Cramér-Rao bound (CRB), which is given as the inverse of the Fisher information matrix (FIM). Often, it is more convenient to express the FIM as

$$
\mathcal{F}\left(\boldsymbol{\theta}_{p}\right)=\int\left(\nabla_{\boldsymbol{\theta}_{p}} \ln p\left(\boldsymbol{r} ; \boldsymbol{\theta}_{p}\right) \nabla_{\boldsymbol{\theta}}^{T} \ln p\left(\boldsymbol{r} ; \boldsymbol{\theta}_{p}\right)\right) p\left(\boldsymbol{r} ; \boldsymbol{\theta}_{p}\right) \mathrm{d} \boldsymbol{r} .
$$

As we are considering a localization problem, position estimation performance is of utmost interest. Hence, we assume that the actual measurement error distribution $p_{V}(v)$ is precisely known when deriving the performance bound. The entries in $\boldsymbol{\theta}_{p}$ are reordered as $\boldsymbol{\theta}_{p}=\left[x_{1}, x_{2}, \ldots, x_{N_{u}}, y_{1}, y_{2}, \ldots, y_{N_{u}}\right]^{T}$ for convenience.

Combining the steps given in [31] and [3], the FIM of $\boldsymbol{\theta}_{p}$ given the true $p_{V}(v)$ is easy to obtain as

$$
\mathcal{F}\left(\boldsymbol{\theta}_{p}\right)=\left(\begin{array}{ll}
\mathcal{F}_{\boldsymbol{x} \boldsymbol{x}} & \mathcal{F}_{\boldsymbol{x} \boldsymbol{y}} \\
\mathcal{F}_{\boldsymbol{x} \boldsymbol{y}}^{T} & \mathcal{F}_{\boldsymbol{y} \boldsymbol{y}}
\end{array}\right)
$$

where $\mathcal{F}_{\boldsymbol{x} \boldsymbol{x}}, \mathcal{F}_{\boldsymbol{x} \boldsymbol{y}}$ and $\mathcal{F}_{\boldsymbol{y} \boldsymbol{y}}$ are all square matrices of dimension $N_{u} \times N_{u}$ and

$$
\left[\mathcal{F}_{\boldsymbol{m} \boldsymbol{n}}\right]_{i, i^{\prime}}=\left\{\begin{array}{cc}
\mathcal{I}_{v} \cdot \sum_{\forall j \in \mathcal{H}(i)} \frac{\left(m_{i}-m_{j}\right)\left(n_{i}-n_{j}\right)}{\left\|\boldsymbol{p}_{i}-\boldsymbol{p}_{j}\right\|^{2}}, & i=i^{\prime} \\
-\mathcal{I}_{v} \cdot \delta_{i, i^{\prime}} \cdot \frac{\left(m_{i}-m_{i^{\prime}}\right)\left(n_{i}-n_{i^{\prime}}\right)}{\left\|\boldsymbol{p}_{i}-\boldsymbol{p}_{i^{\prime}}\right\|^{2}}, & i \neq i^{\prime}
\end{array},\right.
$$

for $\boldsymbol{m}, \boldsymbol{n} \in\{\boldsymbol{x}, \boldsymbol{y}\}$. Here, $\delta_{i, i^{\prime}}$ is Kronecker's delta defined by

$$
\delta_{i, i^{\prime}}= \begin{cases}1, & \text { if } i^{\prime} \in \mathcal{H}(i) \\ 0, & \text { if } i^{\prime} \notin \mathcal{H}(i)\end{cases}
$$

and

$$
\mathcal{I}_{v}=\int \frac{\left[\nabla_{v} p_{V}(v)\right]^{2}}{p_{V}(v)} \mathrm{d} v
$$

The scalar factor $\mathcal{I}_{v}$ is known as the intrinsic accuracy [45] and often approximated via Monte Carlo integration as

$$
\mathcal{I}_{v} \approx \frac{1}{N_{M}} \sum_{n=1}^{N_{M}} \frac{\left[\nabla_{v} p_{V}\left(v^{(n)}\right)\right]^{2}}{p_{V}^{2}\left(v^{(n)}\right)}
$$

where $v^{(n)}, n=1,2, \ldots, N_{M}$ are i.i.d. samples generated from $p_{V}(v)$. In fact, (25) works for any distribution of known form that fulfills the regularity conditions listed in [45]. For the special case $p_{V}(v)=\mathcal{N}\left(v ; \mu_{v}, \sigma_{v}^{2}\right)$, we have $\mathcal{I}_{v}=\sigma_{v}^{-2}$ in closed form and the result in (24) coincides with the one derived for a single Gaussian model in [3]. Finally, the CRB is given by $\operatorname{CRB}\left(\boldsymbol{\theta}_{p}\right) \triangleq \mathcal{F}^{-1}\left(\boldsymbol{\theta}_{p}\right)$.

In the simulations, the localization accuracy is evaluated in terms of the overall localization root-mean-square-error (RMSE), which is defined by

$$
\overline{\mathrm{RMSE}} \triangleq\left(\frac{1}{N_{u}} \sum_{i=1}^{N_{u}} \mathbb{E}\left[\left(\hat{x}_{i}-x_{i}\right)^{2}+\left(\hat{y}_{i}-y_{i}\right)^{2}\right]\right)^{1 / 2}
$$

Assuming that $\left[\hat{x}_{i}, \hat{y}_{i}\right]$ is any unbiased estimator of the true position $\left[x_{i}, y_{i}\right]$, The lower bound of $\overline{\mathrm{RMSE}}$ is given by

$$
\overline{\mathrm{CRB}}_{p o s}\left(\boldsymbol{\theta}_{p}\right) \triangleq \sqrt{\frac{1}{N_{u}} \operatorname{tr}\left[\mathrm{CRB}\left(\boldsymbol{\theta}_{p}\right)\right]} .
$$

\section{RESULTS}

Throughout the simulations, we set $N_{i t r}=N_{i t r}^{E}=75$ ECM iterations, $N_{i t r}^{G}=10$ iterations within every single ECM iteration, and $\Delta_{c}=0.01$ as the convergence tolerance for the centralized algorithms. The measurements are generated such that $r_{i, j}=r_{j, i}$. For comparison purposes, we choose various competing algorithms, including the distributed LS algorithm [5, Algorithm 1] and the classical SPAWN algorithm [5] as well as two parametric variations [15] and [16]. It is well known that the distributed LS algorithm does not require any knowledge about the measurement error statistics. Throughout this section, we assume that all the SPAWN algorithms know the actual mixture model parameters and the underlying channel state (LOS or NLOS state) for every communication link. However, we note that these two assumptions can rarely hold in reality.

Details about the implementation of these competitors are as follows. A distributed LS estimate is found by following the routine given in [5] with the initial guess selected randomly and uniformly from the deployed area. The number of iterations is set to $N_{i t r}=N_{i t r}^{L}=500$ iterations. The classical SPAWN algorithm sets $R_{\text {int }}=R_{\text {bel }}=500$ samples. The first parametric SPAWN algorithm [15] uses a three-mode Gaussian mixture to represent the belief messages and a tenmode Gaussian mixture to represent the internal messages. A second parametric SPAWN algorithm modifies [16] by adopting three circular distributions to represent the internal messages while a three-mode Gaussian mixture to model the belief messages. All SPAWN algorithms terminate after $N_{i t r}=N_{i t r}^{S P}=10$ iterations. All the simulations considered afterwards are performed in MATLAB ${ }^{\mathrm{TM}}$ environment on a PC equipped with Intel $\mathrm{R}$ Core $^{\mathrm{TM}} \mathrm{i} 5-760$ processor $(2.80 \mathrm{GHz})$ and 8GB RAM.

\section{A. Simulation Results: Influence of Network Size}

We consider three stationary networks with $N$ sensors uniformly arranged into $\sqrt{N}$ rows and $\sqrt{N}$ columns in a 40meter $(\mathrm{m})$ by $40-\mathrm{m}$ square area, like in [2, Fig. 6]. In the sequel, the total number of agents, $N_{u}$, takes 45, 96, 192, respectively. The anchors are located in the four corners of the square area. The actual distance measurement error is assumed to follow a two-mode Gaussian mixture distribution with parameters $\alpha_{1}=0.8, \mu_{1}=-0.3 \mathrm{~m}, \sigma_{1}=0.2 \mathrm{~m}$, $\alpha_{2}=0.2, \mu_{2}=4.4 \mathrm{~m}, \sigma_{2}=3.2 \mathrm{~m}$ for a mixed LOS/NLOS environment. The actual mean and variance of each mixture component are set according to an indoor campaign results given in [23]. We further assume a quasi-static environment where the measurement error statistics stays unaltered during the localization process. Throughout this subsection, we do not assume model mismatch between the actual measurement error distribution and the parametric model with $C=2$ in (2). We aim to study how the network size would influence different algorithms in terms of (1) overall localization RMSE (cf. (26)) and (2) computational time (in seconds) and (3) communication overhead (in megabytes). To simplify our comparisons, all the three networks are assumed to be fully connected in the sequel. 
We perform a Monte Carlo experiment with 100 independent trials for each sensor network. An initial guess of the distributed ECM algorithms is determined according to the strategy given in Section III-D. More precisely, we determine $\boldsymbol{\theta}_{m}^{(0)}$ by conducting conventional Gaussian mixture learning upon 50 measurement error samples collected between two anchors. After $\boldsymbol{\theta}_{m}^{(0)}$ is obtained, it will be dispersed via a series of broadcasts to all sensors. An initial guess of the agent positions is determined by running the distributed LS algorithm [5, Algorithm 1]. After each agent obtained a local estimate of its own position, $\boldsymbol{p}_{i}^{(0)}$, it is also broadcast to the neighboring sensors $j, j \in \mathcal{H}(i)$. For fair comparisons, the centralized ECM algorithms use the same initial guess, although better strategies might exist.

We start to evaluate the overall localization RMSE versus the network size, $N$, which takes 49,100, and 196 sensors, respectively. The results are shown along with the performance lower bound $\overline{\mathrm{CRB}}_{\text {pos }}\left(\boldsymbol{\theta}_{p}\right)$ in Fig. 2. It is observed that all the RMSE curves decrease monotonically as $N$ increases. This can be explained from a Fisher information theoretic point of view. Since the sensor networks are assumed to be fully connected, more sensor pairs conduct measurements as $N$ increases, leading to more information about the relative positions.

The RMSE curves of the distributed ECM algorithms are close to those of the centralized counterparts. This is because the local estimates of the Gaussian mixture model parameters calculated through the average consensus algorithm are close to the corresponding global estimate for every single ECM iteration. The results obtained here have shown that a centralized ECM algorithm slightly outperforms its distributed approximation in terms of the overall localization RMSE. However, this is not guaranteed for all cases. For instance, we have observed under other settings (cf. Section VI-C) that a distributed ECM algorithm can generate even lower RMSE. One possible reason is that a distributed ECM estimator is biased in essence due to the approximations introduced therein. Besides, the corresponding centralized ECM estimator might be biased as well due to convergence to local optimum or insufficient number of iterations. Generally speaking, the 1-D GS based ECM algorithms are inferior to the 2-D BFGS-QN based algorithms due to the discretization of the solution space by a finite number of grids, for instance $R_{\text {grid }}=150$ used here. Enlarging $R_{\text {grid }}$ can effectively alleviate this problem, however, at the cost of higher computational cost.

In Fig. 2, it is observed that the ECM algorithms perform closely to the classical SPAWN algorithm configured with perfect environmental knowledge and outperform the distributed LS algorithm by far. ${ }^{4}$ It is also remarkable that the ECM algorithms clearly outperform the parametric SPAWN algorithm [16] when $N \geq 100$. For smaller $N$, for instance 49 considered here, the localization RMSE of the ECM algorithms can deviate from the performance lower bound. Possible reasons are as follows. Firstly, more ECM iterations

\footnotetext{
${ }^{4}$ Due to the high computational complexity, the classical SPAWN algorithm has been tested only for smaller network sizes, $N=49$ and $N=100$ herein, on the Lichtenberg-high performance computer of TU Darmstadt, Germany.
}

are needed to converge. Secondly, the ECM algorithm more often get stuck at local optimum. Thirdly, the total number of measurements are still insufficient for the ECM algorithms to demonstrate the asymptotic properties. As a conclusion, the proposed ECM algorithms are less suitable to use for the networks of rather small size, which is similar to applying the conventional EM algorithm for Gaussian mixture learning with only insufficient number of measurements.

As a complement of the theoretical computational complexity analysis given in Section IV-A, we next evaluate the average computational time for a single agent to run one complete trial of different distributed algorithms. Here, we also take into account the influence of the number of iterations $N_{i t r}$. The corresponding design parameters for this experiment are $N_{i t r}=N_{i t r}^{L}=500$ for the distributed LS algorithm, $R_{b e l}=500$ samples and $N_{i t r}=N_{i t r}^{S P}=10$ for the classical SPAWN algorithm, $R_{i n t}=500$ samples and $N_{i t r}=N_{i t r}^{S P}=$ 10 for the modified parametric SPAWN algorithm [16], $C=2$, $N_{i t r}=N_{i t r}^{E}=75, N_{i t r}^{G}=10, N_{i t r}^{n u} \sim 10^{1}-10^{2}$, and $R_{\text {grid }}=150$ for the distributed ECM algorithms. The results are shown as a function of the network size, $N$, in the top subfigure of Fig. 3. The results show that the distributed ECM algorithms are inferior to the distributed LS algorithm but outperform the parametric SPAWN algorithm by far in terms of the average computational time. It is noteworthy that the computational time stays nearly unaltered rather than increases linearly in terms of $N$ for the distributed LS algorithm. The reason is that in the distributed LS algorithm we adopt a simple censoring strategy to make the number of neighboring sensors, $|\mathcal{H}(i)|$, always equal to a small fixed number rather than $N-1$ for the assumed fully connected networks. In this way, the distributed LS algorithm is less complex to use for initializing the proposed ECM algorithms.

Next, communication overhead is further evaluated in terms of the amount of megabytes (MB) consumed (for broadcast) by all sensors to run one complete trial of different distributed algorithms. The total number of MB consumed by the distributed ECM algorithms is $6 N_{i t r}^{E} N_{u} N_{i t r}^{G} C / 2^{17} \approx 0.07 N_{u} \mathrm{MB}$. The total number of MB consumed by the classical SPAWN algorithm is $3 R_{b e l} N_{i t r}^{S P} N_{u} / 2^{17} \approx 0.12 N_{u} \mathrm{MB}$, where the constant factor 3 comes from the fact that each particle contains two real values representing a candidate position in 2-D space and has a real weighting factor. The total number of MB consumed by the modified parametric SPAWN algorithm [16] is equally reduced to $C_{b e l} N_{i t r}^{S P} N_{u} / 2^{17} \approx 0.001 N_{u} \mathrm{MB}$ due to the use of a parametric model. Note that $C_{b e l}$ is equal to 17 in the above expression, denoting the total number of real values broadcast by each agent for one iteration. This is because a three mode Gaussian mixture is used to represent the belief message, and each agent needs to broadcast two real values for the mixing coefficients, 6 real values for the mean vectors, and 9 real values for the symmetric covariance matrices. The total number of MB that the distributed LS algorithm consumed is $2 N_{i t r}^{L} N_{u} / 2^{17} \approx 0.008 N_{u} \mathrm{MB}$. This is because each agent needs to broadcast only two real values of its own position estimate on each iteration in the distributed LS algorithm. The above theoretical results are depicted as a function of $N_{u}$ in the bottom sub-figure of Fig. 3. It is quite obvious 


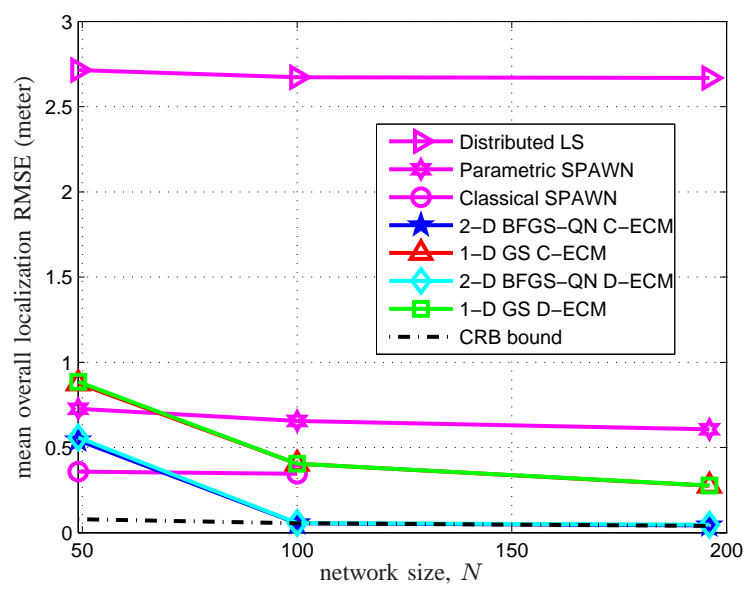

Fig. 2. Mean overall localization RMSE of different cooperative localization algorithms as a function of the network size, $N$.
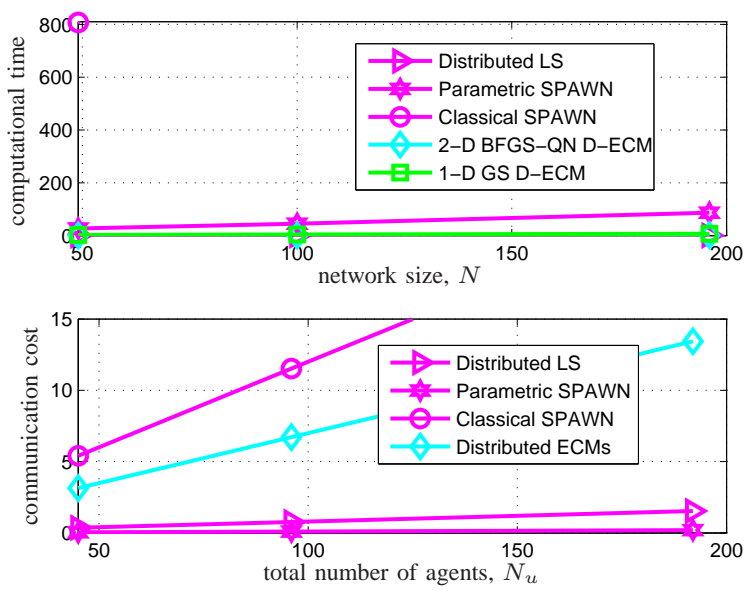

Fig. 3. Top sub-figure: Average computational time of different distributed algorithms as a function of the network size, $N$. Bottom sub-figure: Total communication cost of different distributed algorithms as a function of the number of agents, $N_{u}$.

that the classical SPAWN algorithm needs the largest amount of energy for wireless communications, followed by the distributed LS algorithm, the parametric SPAWN algorithm, and the distributed ECM algorithms. Part of the results obtained here contradict those in Section IV-B but are not surprising, because the influence of $N_{i t r}$ has been taken into account. More precisely, $N_{i t r}^{S P}$ is set very small as compared to $N_{i t r}^{L}$ and $N_{i t r}^{E}$, hence the parametric SPAWN algorithm is superior to the distributed LS algorithm and ECM algorithms in the above experiment. ${ }^{5}$

\section{B. Simulation Results: Varying Measurement Error Statistics}

In this subsection, we re-evaluate the overall localization RMSE for the distributed ECM algorithms using different network topologies and varying measurement error statistics. Each network consists of 105 sensors, among which 100

\footnotetext{
${ }^{5}$ Herein, the SPAWN algorithms are not ensured to converge but can achieve reasonably good result after a few (usually less than 20) iterations.
}

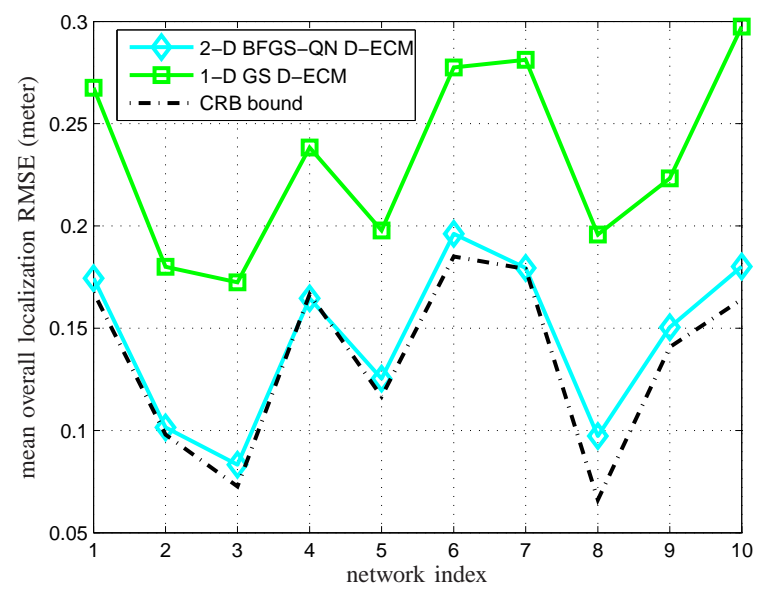

Fig. 4. Mean overall localization RMSE of two distributed ECM algorithms for different sensor network topologies and measurement error distributions.
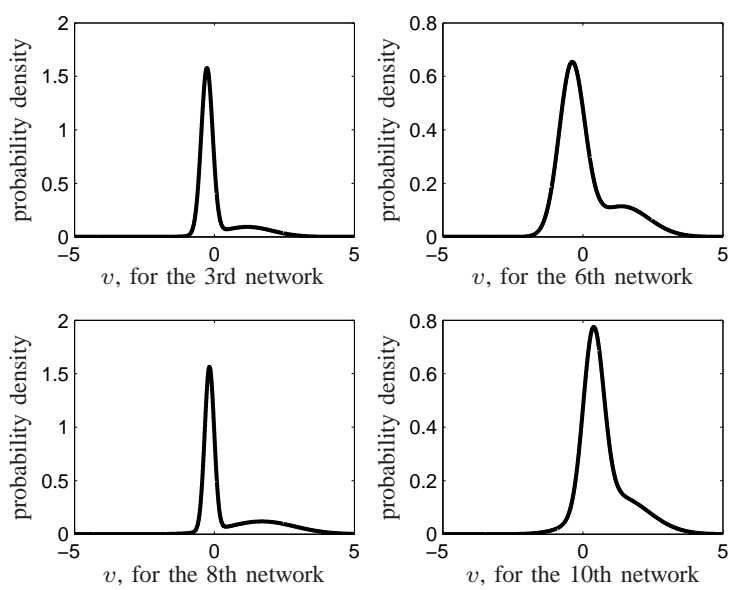

Fig. 5. Actual measurement error distributions for different network topologies. Overlap of the Gaussian mixture components is assumed.

agents and 5 anchors. The agent positions are now generated uniformly from a $15 \mathrm{~m} \times 15 \mathrm{~m}$ square area. The five anchors are placed in the four corners and the center of the deployed area. The communication range $R_{c}$ is set to 10 meter. In total ten different sensor networks are generated for test, and for each network the measurement error is assumed to follow a two-mode Gaussian mixture but with randomized parameters. More precisely, $\alpha_{1}$ is uniformly generated from $\mathcal{U}[0.6,0.8), \mu_{1}$ from $\mathcal{U}[-0.5,0.5), \sigma_{1}$ from $\mathcal{U}[0.1,0.5), \mu_{2}$ from $\mathcal{U}[0.8,1.8)$, and $\sigma_{2}$ from $\mathcal{U}[0.8,1.2)$. The mean and standard deviation are measured in meters. Contrary to the measurement error distribution used in Section VI-A, the two Gaussian mixture components are now partly overlapping. The two distributed ECM algorithms assume $C=2$ in the parametric measurement error model and use the same initialization strategy as introduced before. In order to provide better localization performance, the 1-D GS D-ECM algorithm adopts $R_{\text {grid }}=400$.

The RMSE curves are shown together with the performance bound for each sensor network in Fig. 4. In Fig. 5, we further 
show the actual measurement error distribution respectively for the 3rd, 6th, 8th, and 10th sensor network, with which more obvious performance degradation can be observed. Although the results do not demonstrate clear performance drop, we note that the overlap of Gaussian mixture components may result in degraded performance. The reasons are the following. Firstly, an initial guess of the Gaussian mixture model parameters might be erroneously determined due to the misclassification of some measurements (especially those generated from the overlapping area), which will make the ECM algorithms converge to some unwanted result, like saddle point. Secondly, the ECM algorithms might wrongly converge to some position estimate such that the residuals can be very well classified into $C$ clusters. Thirdly, convergence speed of the ECM algorithms might become slower.

\section{Experimental Results}

Lastly, we adopt the real sensor network and TOA measurements described at the beginning of [3, Section IV]. The setup is shortly reviewed as follows. The measurement environment is a typical office area located at the Motorola facility in the USA. The network consists of 44 sensors in total, among which 40 agents and 4 anchors. The measurement system uses a wideband direct-sequence spread-spectrum (DS-SS) transmitter and receiver (Sigtek model ST-515). High SNR was maintained throughout the measurement campaign.

The distance measurement error after bias remedy $(\mu=$ $10.9 \mathrm{~ns} \times c \approx 3.26 \mathrm{~m}$ ) was justified via Kolmogorov-Smirnov (KS) test to well fit a Gaussian distribution with zero mean and standard deviation $\sigma=6.1 \mathrm{~ns} \times c \approx 1.83 \mathrm{~m}$, although the histogram manifests slightly skewed and heavy tailed profile as will be seen later. In practice, however, bias remedy can hardly be done as in [3] for lack of the actual agent positions. To be as realistic as possible, the proposed ECM algorithms use the original distance measurements (without bias remedy) and further assume unknown knowledge of the underlying measurement error distribution. But their competitors all use the bias remedied data. Moreover, the SPAWN algorithms assume a single Gaussian error model with precisely known mean and variance.

Since unknown knowledge of the measurement error distribution is assumed, we test the ECM algorithms with two different $C$ values, namely $C=1$ (model mismatch free) and $C=3$ (model mismatch). For the case $C=1$, the distributed ECM algorithms only need an initial guess of the agent positions, which can be computed via the distributed LS algorithm. Herein, the distributed LS algorithm is applied to the original data. As will be seen later in Table III, the existence of a bias term in the original data can largely degrade the distributed LS algorithm. For the case $C=3$, the distributed ECM algorithms need also an initial guess of the Gaussian mixture model parameters. As opposed to the previous subsections where $\boldsymbol{\theta}_{m}^{(0)}$ was determined by two communicating anchors, we only coarsely set $\alpha_{1}^{(0)}=0.1, \mu_{1}^{(0)}=0 \mathrm{~m}, \sigma_{1}^{(0)}=\sqrt{3} \mathrm{~m}, \alpha_{2}^{(0)}=0.8, \mu_{2}^{(0)}=$ $3.5 \mathrm{~m}, \sigma_{2}^{(0)}=\sqrt{2} \mathrm{~m}, \alpha_{3}^{(0)}=0.1, \mu_{3}^{(0)}=7 \mathrm{~m}, \sigma_{3}^{(0)}=\sqrt{3} \mathrm{~m}$ herein. ${ }^{6}$ Similarly, the centralized ECM algorithms use the same initial guess for comparison purposes.

The overall localization RMSE is evaluated for different distributed algorithms in a fully connected network. The results are shown in Table III with an extra column indicating which data set is used by a specific algorithm. For better visualization, the estimated agent positions obtained from the two distributed ECM algorithms are depicted for the case $C=3$ in Fig. 6 along with the true sensor positions. Similar results are also obtained for the case $C=1$ but not shown here due to space constraints. In order to shed some light on how tolerant the distributed ECM algorithms are to the initial guess, we depict in Fig. 7 the agent positions estimated by the distributed LS algorithm using the original (bias retained) data set.

TABLE III

LOCALIZATION PERFORMANCE OF DIFFERENT ALGORITHMS

\begin{tabular}{|l|c|c|}
\hline Cooperative Localization Algorithms & RMSE & Data Set \\
\hline Distributed LS [5] & $1.614 \mathrm{~m}$ & bias remedied \\
\hline Distributed LS [5] & $5.160 \mathrm{~m}$ & bias retained \\
\hline Classical SPAWN [5] & $1.146 \mathrm{~m}$ & bias remedied \\
\hline Parametric SPAWN [15] & $1.163 \mathrm{~m}$ & bias remedied \\
\hline Parametric SPAWN [16] & $1.260 \mathrm{~m}$ & bias remedied \\
\hline 2-D BFGS-QN C-ECM $(C=1)$ & $1.387 \mathrm{~m}$ & bias retained \\
\hline 1-D GS C-ECM $(C=1)$ & $1.399 \mathrm{~m}$ & bias retained \\
\hline 2-D BFGS-QN D-ECM $(C=1)$ & $1.393 \mathrm{~m}$ & bias retained \\
\hline 1-D GS D-ECM $(C=1)$ & $1.390 \mathrm{~m}$ & bias retained \\
\hline 2-D BFGS-QN C-ECM $(C=3)$ & $1.402 \mathrm{~m}$ & bias retained \\
\hline 1-D GS C-ECM $(C=3)$ & $1.411 \mathrm{~m}$ & bias retained \\
\hline 2-D BFGS-QN D-ECM $(C=3)$ & $1.405 \mathrm{~m}$ & bias retained \\
\hline 1-D GS D-ECM $(C=3)$ & $1.461 \mathrm{~m}$ & bias retained \\
\hline
\end{tabular}

Although only one set of measurement data is available, the results obtained above reveal the following. Firstly, an unknown bias term in the measurement data can degrade the distributed LS algorithm, or broadly speaking any LS estimation based algorithm. Secondly, it is not surprising to see that the SPAWN algorithms provide the best performance because they have all the necessary information about the measurement error statistics and the assumed uniform prior distribution (for the unknown agent positions) is apparently a good choice. Thirdly, the centralized ECM algorithms with $C=1$ are superior to those with $C=3$, because the former are free of model mismatch. In contrast to the SPAWN algorithms, however, they need to estimate one extra set of mean and variance parameters jointly with the unknown positions. And in contrast to the distributed LS algorithm, they tend to find a better parameter estimate in the sense of maximizing the log-likelihood function $\mathcal{L}(\boldsymbol{\theta} ; \boldsymbol{r})$ rather than converge to some suboptimal solution, for instance the actual agent positions and the calibrated mean and variance [3]. An additional comparison between the estimated Gaussian distribution and the calibrated Gaussian distribution [3] is shown in Fig. 8. Therein, a diffusion based kernel density estimate (KDE) [46]

${ }^{6}$ The reasons for empirically selecting the initial guess are the following. Firstly, the measurement data are close to Gaussian as shown in Fig. 8 of the old manuscript. Therefore, it is hard to get an initial guess of a threemode Gaussian mixture merely from the data collected between the anchors. Secondly, the number of the measurements collected by the anchors might be insufficient for Gaussian mixture learning. 


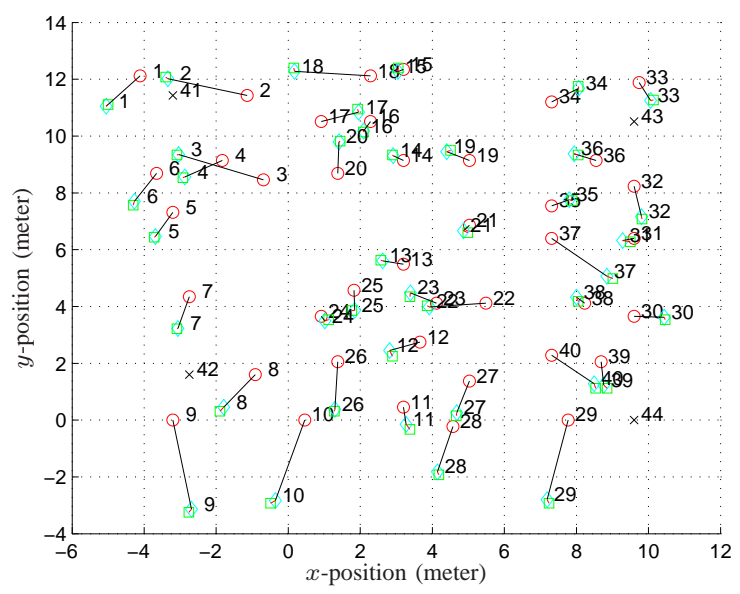

Fig. 6. Estimated agent positions versus the true positions in 2-D plane. Herein, black $\times$ 's denote the anchor positions; red o's denote the true agent positions; cyan $\diamond$ 's denote the agent positions estimated by the 2-D BFGS-QN D-ECM algorithm (with $C=3$ and RMSE $=1.405 \mathrm{~m}$ ); green $\square$ 's denote the agent positions estimated from the 1-D GS D-ECM algorithm (with $C=3$ and RMSE $=1.461 \mathrm{~m}$ ); black -'s represent the localization errors between the true positions and the positions estimated by the 2-D BFGS-QN D-ECM algorithm.

of the actual measurement error distribution is also depicted, which serves as the ground truth. Fourthly, the centralized ECM algorithms with $C=3$ degraded only slightly, as the final PDF estimate (essentially a three mode Gaussian mixture) degenerated upon convergence almost to the Gaussian PDF estimate obtained for the case $C=1$. This can be seen clearly from Fig. 8. Therein, it is also observed that the modes of these two PDF estimates are very close to that of the KDE. This is critical for an unbiased estimator to be found by the proposed centralized algorithms. Fifthly, the distributed ECM algorithms perform closely to their centralized counterparts, and the explanations have been given before. Sixthly, the ECM algorithms show good tolerance to an initial guess.

The main purposes of this section are twofold. On the one hand, the ECM algorithms were tested with and without model mismatch. On the other hand, different cooperative localization algorithms were tested with one set of real measurement data. We also stress that the comparison of different algorithms is not our focus here, since particularly only one set of data is available.

\section{CONCLUSION}

In this paper, we have proposed a series of expectationconditional maximization (ECM) algorithms for cooperative localization in non-Gaussian measurement errors approximated by finite-mode Gaussian mixture. The centralized ECM algorithms have been proven under some conditions to be able to increase the incomplete data log-likelihood monotonically towards a stationary value. Distributed ECM algorithms have also been developed to resolve the scalability problem for large-scale sensor networks. Systematical analyses have shown that the proposed distributed algorithms perform analogously to the parametric SPAWN algorithms in terms of both computational complexity and communication overhead. Simulation-

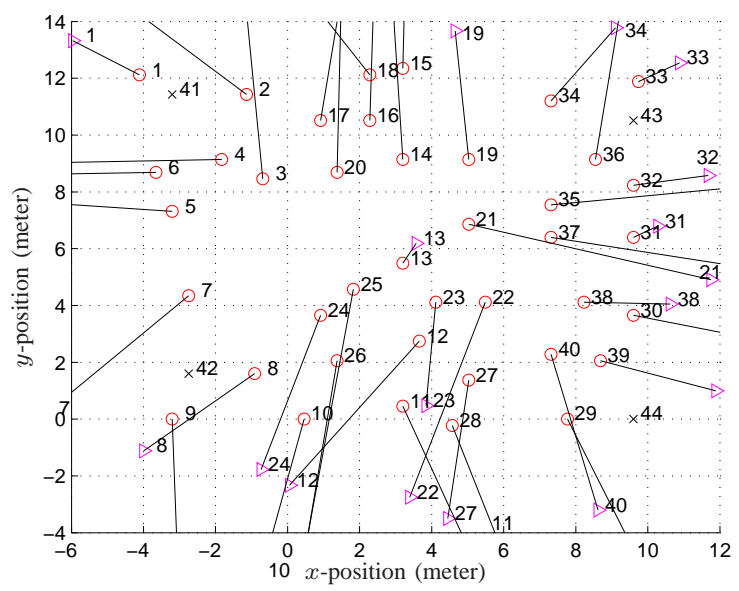

Fig. 7. Estimated agent positions versus the true positions in 2-D plane. Herein, black $\times$ 's denote the anchor positions; red o's denote the true agent positions; magenta $\triangleright$ 's denote the agent positions estimated by the distributed LS algorithm; black -'s represent the localization errors between the true positions and the estimated positions. Note that, the distributed LS algorithm uses the original (bias retained) data set.
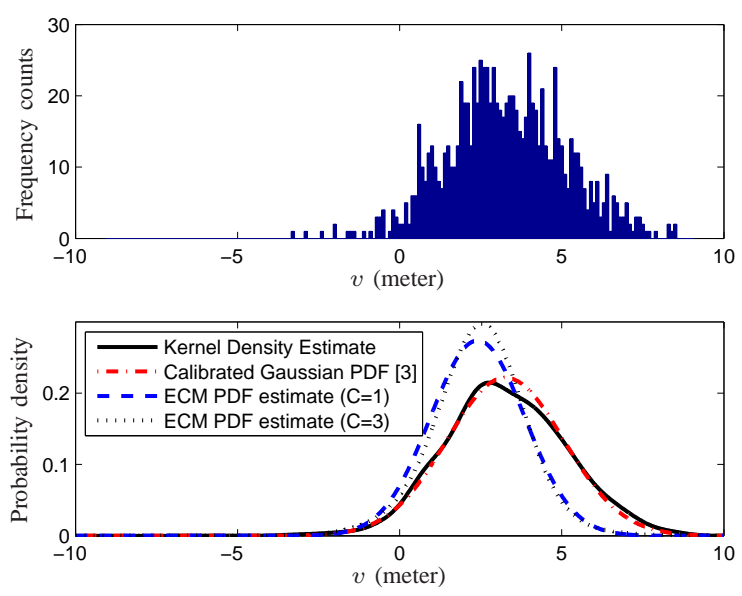

Fig. 8. Top sub-figure: histogram of the actual measurement error residuals $v_{i, j}, \forall(i, j) \in \Gamma$ for $R_{c}=\infty$ meter. Bottom sub-figure: different distributive profiles (including the offline calibrated Gaussian PDF [3], two PDF estimates obtained for $C=1$ (with $\hat{\mu}_{1}=2.404$ and $\hat{\sigma}_{1}^{2}=2.123$ ) and $C=3$ (with $\hat{\alpha}_{1}=0.181, \hat{\mu}_{1}=1.319, \hat{\sigma}_{1}^{2}=2.661$ and $\hat{\alpha}_{2}=0.796$, $\hat{\mu}_{2}=2.619, \hat{\sigma}_{2}^{2}=1.447$ and $\hat{\alpha}_{3}=0.024, \hat{\mu}_{3}=5.422, \hat{\sigma}_{3}^{2}=1.691$ ) respectively from the 2-D BFGS-QN C-ECM algorithm) versus a diffusion based kernel density estimate [46], which serves as the underlying distribution for lack of ground truth.

and experimental results have demonstrated that: (1) the proposed ECM algorithms nearly attain the performance lower bound when the data size is much larger than the number of unknowns and free of model mismatch; (2) the proposed algorithms can work properly but sub-optimality under model mismatch. The proposed ECM algorithms are non-Bayesian in nature. They are more robust against outliers than the conventional LS algorithms. In contrast to some Bayesian algorithms, for instance the SPAWN algorithms, they require neither precise measurement error statistics nor complicated NLOS identification. Therefore, they are suitable for a wider range of WSN applications. Nevertheless, many research chal- 
lenges need to be met in our future work, including (1) reduction of computational complexity and communication overhead; (2) consideration of lossy wireless transmission and quantized messages.

\section{APPENDIX A}

\section{PROOF OF THEOREM 1}

The result given in Remark 2 reveals that the proposed centralized ECM algorithms can converge monotonically to some value $L^{*}$ of the incomplete-data log-likelihood function $\mathcal{L}(\boldsymbol{\theta} ; \boldsymbol{r})$ when bounded above. For $L^{*}$ to be a stationary point, however, we need to further prove, according to [29], that: (1) $g_{s}(\boldsymbol{\theta})$ is differentiable; (2) the corresponding gradient $\nabla_{\boldsymbol{\theta}} g_{s}(\boldsymbol{\theta})$ is of full rank at $\boldsymbol{\theta}^{(\eta)} \in \Theta$, for all $\eta$; and (3) the "space filling" condition holds as

$$
\bigcap_{s=1}^{S} G_{s}\left(\boldsymbol{\theta}^{(\eta)}\right)=\{\mathbf{0}\}, \quad \text { for all } \eta,
$$

where $G_{s}(\boldsymbol{\theta})$ is the column space of the matrix $\nabla_{\boldsymbol{\theta}} g_{s}(\boldsymbol{\theta})$. Due to space limitations, we only give the proof for the 1-D GS C-ECM algorithm. Similar methodology applies to the 2-D BFGS-QN C-ECM algorithm.

In the 1-D GS C-ECM algorithm, we have $\operatorname{dim}(\boldsymbol{\theta})=3 C+$ $2 N_{u}$ and $S=2 N_{u}+2 C+1$. It is easy to show that

$$
\begin{aligned}
& \nabla_{\boldsymbol{\theta}} g_{s}\left(\boldsymbol{\theta}^{(\eta)}\right)= \\
& \begin{cases}{\left[\mathbf{e}_{C+1}, \ldots, \mathbf{e}_{\operatorname{dim}(\boldsymbol{\theta})}\right],} & s=1 \\
{\left[\mathbf{e}_{1}, \ldots, \mathbf{e}_{C+s-2}, \mathbf{e}_{C+s}, \ldots, \mathbf{e}_{\operatorname{dim}(\boldsymbol{\theta})}\right],} & s=2, \ldots, S-1 \\
{\left[\mathbf{e}_{1}, \ldots, \mathbf{e}_{3 C+2 N_{u}-1}\right],} & s=S\end{cases}
\end{aligned}
$$

is differentiable and irrespective of $\boldsymbol{\theta}^{(\eta)}$ because

$$
\mathbf{e}_{j} \triangleq[\underbrace{0, \ldots, 0}_{j-1 \text { copies }}, 1, \underbrace{0, \ldots, 0}_{\operatorname{dim}(\boldsymbol{\theta})-j \text { copies }}]^{T}, \quad \forall j \in\{1,2, \ldots, \operatorname{dim}(\boldsymbol{\theta})\} .
$$

It is clear that $\nabla_{\boldsymbol{\theta}} g_{1}\left(\boldsymbol{\theta}^{(\eta)}\right)$ is of dimension $\operatorname{dim}(\boldsymbol{\theta}) \times(\operatorname{dim}(\boldsymbol{\theta})-$ $C)$; and $\nabla_{\boldsymbol{\theta}} g_{s}\left(\boldsymbol{\theta}^{(\eta)}\right), s=2, \ldots, S$, are all of dimension $\operatorname{dim}(\boldsymbol{\theta}) \times(\operatorname{dim}(\boldsymbol{\theta})-1)$. Since $\mathbf{e}_{j}$ and $\mathbf{e}_{j^{\prime}}$ are mutually orthogonal for $j \neq j^{\prime}, \nabla_{\boldsymbol{\theta}} g_{s}\left(\boldsymbol{\theta}^{(\eta)}\right)$ owns a full column rank for any $s$. So far, the first two conditions have been proven. In the sequel, we omit the ECM iteration index $\eta$ for brevity.

The proof of the third condition starts with the definition of column space, that is, $G_{s}(\boldsymbol{\theta})$ is a linear combination of the columns of the matrix $\nabla_{\boldsymbol{\theta}} g_{s}(\boldsymbol{\theta})$, i.e.,

$$
G_{s}(\boldsymbol{\theta})= \begin{cases}\sum_{j=\{C+1, \ldots, \operatorname{dim}(\boldsymbol{\theta})\}} c_{j} \mathbf{e}_{j}, & s=1 \\ \sum_{j=\{1, \ldots, \operatorname{dim}(\boldsymbol{\theta})\} \backslash\{C+s-1\}} c_{j} \mathbf{e}_{j}, & s=2, \ldots, S\end{cases}
$$

where $c_{j}$ is any real non-zero scalar coefficient. Since $G_{s}(\boldsymbol{\theta})$ is a subspace of $\mathbb{R}^{\operatorname{dim}(\boldsymbol{\theta})}$, (27) can be reformulated as

$$
\begin{aligned}
\bigcap_{s=1}^{S} G_{s}(\boldsymbol{\theta}) & =\bigcap_{s=0}^{S} G_{s}(\boldsymbol{\theta}) \\
& =G_{S}(\boldsymbol{\theta}) \bigcap G_{S-1}(\boldsymbol{\theta}) \bigcap \cdots \bigcap G_{1}(\boldsymbol{\theta}) \bigcap G_{0}(\boldsymbol{\theta})
\end{aligned}
$$

where $G_{0}(\boldsymbol{\theta})$ is the whole space of $\mathbb{R}^{\operatorname{dim}(\boldsymbol{\theta})}$, spanned by $\sum_{j=\{1, \ldots, \operatorname{dim}(\boldsymbol{\theta})\}} c_{j} \mathbf{e}_{j}$. The right-hand-side of the second equation in (29) can be performed sequentially in the order $s=1,2, \ldots, S$, more precisely,

$$
\tilde{G}_{s}(\boldsymbol{\theta})=G_{s}(\boldsymbol{\theta}) \bigcap \tilde{G}_{s-1}(\boldsymbol{\theta})
$$

where

$$
\tilde{G}_{s-1}(\boldsymbol{\theta}) \triangleq G_{s-1}(\boldsymbol{\theta}) \bigcap G_{s-2}(\boldsymbol{\theta}) \bigcap \cdots \bigcap G_{1}(\boldsymbol{\theta}) \bigcap G_{0}(\boldsymbol{\theta})
$$

for $s>1$ while $\tilde{G}_{s-1}(\boldsymbol{\theta})=G_{0}(\boldsymbol{\theta})$ for $s=1$. Starting from $s=1$, we obtain, owing to the dimension formula [47], that

$$
\begin{aligned}
\operatorname{dim} & \left(\tilde{G}_{1}(\boldsymbol{\theta})\right) \\
& =\operatorname{dim}\left(G_{1}(\boldsymbol{\theta}) \bigcap G_{0}(\boldsymbol{\theta})\right) \\
& =\operatorname{dim}\left(G_{1}(\boldsymbol{\theta})\right)+\operatorname{dim}\left(G_{0}(\boldsymbol{\theta})\right)-\operatorname{dim}\left(G_{1}(\boldsymbol{\theta})+G_{0}(\boldsymbol{\theta})\right) .
\end{aligned}
$$

It is easy to see that $\operatorname{dim}\left(G_{1}(\boldsymbol{\theta})\right)=\operatorname{dim}(\boldsymbol{\theta})-C$ and $G_{0}(\boldsymbol{\theta})=$ $\operatorname{dim}(\boldsymbol{\theta})$. The dimension of the sum of the two column spaces, $\operatorname{dim}\left(G_{1}(\boldsymbol{\theta})+G_{0}(\boldsymbol{\theta})\right)$, is equal to the rank of the augmented matrix $\left[\mathbf{e}_{C+1}, \ldots, \mathbf{e}_{\operatorname{dim}(\boldsymbol{\theta})} \mid \mathbf{e}_{1}, \mathbf{e}_{2}, \ldots, \mathbf{e}_{\operatorname{dim}(\boldsymbol{\theta})}\right]$, namely $\operatorname{dim}(\boldsymbol{\theta})$. As a consequence, we obtain $\operatorname{dim}\left(\tilde{G}_{1}(\boldsymbol{\theta})\right)=\operatorname{dim}(\boldsymbol{\theta})-C$. The basis vectors required to span $\tilde{G}_{1}(\boldsymbol{\theta})$ are just the column vectors that $G_{1}(\boldsymbol{\theta})$ and $G_{0}(\boldsymbol{\theta})$ have in common, namely,

$$
\tilde{G}_{1}(\boldsymbol{\theta})=G_{1}(\boldsymbol{\theta}) \bigcap G_{0}(\boldsymbol{\theta})=\sum_{j \in\{C+1, \ldots, \operatorname{dim}(\boldsymbol{\theta})\}} c_{j} \mathbf{e}_{j} .
$$

Similarly for $s>1$, we have

$$
\begin{aligned}
\operatorname{dim} & \left(\tilde{G}_{s}(\boldsymbol{\theta})\right) \\
& =\operatorname{dim}\left(G_{s}(\boldsymbol{\theta}) \bigcap \tilde{G}_{s-1}(\boldsymbol{\theta})\right) \\
& =(\operatorname{dim}(\boldsymbol{\theta})-1)+(\operatorname{dim}(\boldsymbol{\theta})-C-(s-2))-\operatorname{dim}(\boldsymbol{\theta}) \\
& =\operatorname{dim}(\boldsymbol{\theta})-C-s+1,
\end{aligned}
$$

and

$$
\tilde{G}_{s}(\boldsymbol{\theta})=G_{s}(\boldsymbol{\theta}) \bigcap \tilde{G}_{s-1}(\boldsymbol{\theta})=\sum_{j \in\{C+s, \ldots, \operatorname{dim}(\boldsymbol{\theta})\}} c_{j} \mathbf{e}_{j} .
$$

Note that the result derived in (30) is due to the fact that the dimension of $G_{s}(\boldsymbol{\theta})$ is always equal to $\operatorname{dim}(\boldsymbol{\theta})-1$ for $s>1$ and the dimension of the sum of the column spaces, $\operatorname{dim}\left(G_{s}(\boldsymbol{\theta})+\tilde{G}_{s-1}(\boldsymbol{\theta})\right)$, is always equal to $\operatorname{dim}(\boldsymbol{\theta})$. Finally, after the $S$ th intersection, we have

$$
\tilde{G}_{S}(\boldsymbol{\theta})=G_{S}(\boldsymbol{\theta}) \bigcap \tilde{G}_{S-1}(\boldsymbol{\theta})=\{\mathbf{0}\} .
$$

\section{APPENDIX B}

Centralized Parameter Estimates Computed by Assuming $r_{i, j} \neq r_{j, i}$ AND Using All Measurements

We re-consider the two partitions of $\boldsymbol{\theta}$ shown in Example 1 and Example 2 of Section III-B. The centralized (global) estimate of $\alpha_{l}, \mu_{l}$, and $\sigma_{l}^{2}$ on the $(\eta+1)$ th iteration can be easily found to be

$$
\alpha_{l}^{(\eta+1)}=\frac{1}{|\Gamma|} \sum_{i=1}^{N} \sum_{j \in \mathcal{H}(i)} P_{l, i, j}^{(\eta)}
$$




$$
\begin{gathered}
\mu_{l}^{(\eta+1)}=\frac{\sum_{i=1}^{N} \sum_{j \in \mathcal{H}(i)}\left(r_{i, j}-d\left(\boldsymbol{p}_{i}^{(\eta)}, \boldsymbol{p}_{j}^{(\eta)}\right)\right) P_{l, i, j}^{(\eta)}}{|\Gamma| \alpha_{l}^{(\eta+1)}}, \\
\sigma_{l}^{2,(\eta+1)}=\frac{\sum_{i=1}^{N} \sum_{j \in \mathcal{H}(i)}\left(r_{i, j}-d\left(\boldsymbol{p}_{i}^{(\eta)}, \boldsymbol{p}_{j}^{(\eta)}\right)\right)^{2} P_{l, i, j}^{(\eta)}}{|\Gamma| \alpha_{l}^{(\eta+1)}}-\left(\mu_{l}^{(\eta+1)}\right)^{2} .
\end{gathered}
$$

For the 2-D BFGS-QN D-ECM algorithm (corresponds to the partition of $\boldsymbol{\theta}$ in Example 1), the position estimate of agent $i$ is updated through

$$
\begin{aligned}
\boldsymbol{p}_{i}^{(\eta+1)}=\underset{\boldsymbol{p}_{i}}{\arg \min } \sum_{j \in \mathcal{H}(i)} & \sum_{l=1}^{C} \frac{\left(r_{i, j}-d\left(\boldsymbol{p}_{i}, \boldsymbol{p}_{j}^{(\tilde{\eta})}\right)-\mu_{l}^{(\eta+1)}\right)^{2}}{\sigma_{l}^{2,(\eta+1)} / P_{l, i, j}^{(\eta)}} \\
& +\frac{\left(r_{j, i}-d\left(\boldsymbol{p}_{i}, \boldsymbol{p}_{j}^{(\tilde{\eta})}\right)-\mu_{l}^{(\eta+1)}\right)^{2}}{\sigma_{l}^{2,(\eta+1)} / P_{l, j, i}^{(\eta)}} .
\end{aligned}
$$

While for the 1-D GS D-ECM algorithm (corresponds to the partition of $\boldsymbol{\theta}$ in Example 2), the position estimate of agent $i$ is updated through

$$
\begin{array}{r}
x_{i}^{(\eta+1)}= \\
\underset{x_{i}}{\arg \min } \sum_{j \in \mathcal{H}(i)} \sum_{l=1}^{C} \frac{\left(r_{i, j}-d\left(\left[x_{i}, y_{i}^{(\eta)}\right]^{T}, \boldsymbol{p}_{j}^{(\tilde{\eta})}\right)-\mu_{l}^{(\eta+1)}\right)^{2}}{\sigma_{l}^{2,(\eta+1)} / P_{l, i, j}^{(\eta)}} \\
+\frac{\left(r_{j, i}-d\left(\left[x_{i}, y_{i}^{(\eta)}\right]^{T}, \boldsymbol{p}_{j}^{(\tilde{\eta})}\right)-\mu_{l}^{(\eta+1)}\right)^{2}}{\sigma_{l}^{2,(\eta+1)} / P_{l, j, i}^{(\eta)}}, \\
y_{i}^{(\eta+1)}=\quad \\
\underset{y_{i}}{\arg \min } \sum_{j \in \mathcal{H}(i)} \sum_{l=1}^{C} \frac{\left(r_{i, j}-d\left(\left[x_{i}^{(\eta+1)}, y_{i}\right]^{T}, \boldsymbol{p}_{j}^{(\tilde{\eta})}\right)-\mu_{l}^{(\eta+1)}\right)^{2}}{\sigma_{l}^{2,(\eta+1)} / P_{l, i, j}^{(\eta)}} \\
+\frac{\left(r_{j, i}-d\left(\left[x_{i}^{(\eta+1)}, y_{i}\right]^{T}, \boldsymbol{p}_{j}^{(\tilde{\eta})}\right)-\mu_{l}^{(\eta+1)}\right)^{2}}{\sigma_{l}^{2,(\eta+1)} / P_{l, j, i}^{(\eta)}} .
\end{array}
$$

\section{REFERENCES}

[1] I. F. Akyildiz, W. Su, Y. Sankarasubramaniam, and E. Cayirci, "Wireless sensor networks: a survey," Comput. Networks J., vol. 38, pp. 393-422, Mar. 2002.

[2] N. Patwari, J. N. Ash, S. Kyperountas, A. O. Hero III, R. L. Moses, and N. S. Correal, "Locating the nodes: cooperative localization in wireless sensor networks," IEEE Signal Process. Mag., vol. 22, no. 4, pp. 54-69, Jul. 2005.

[3] N. Patwari, A. O. Hero III, M. Perkins, N. S. Correal, and R. J. O’Dea, "Relative location estimation in wireless sensor networks," IEEE Trans. Signal Process., vol. 51, no. 8, pp. 2137-2148, Aug. 2003.

[4] X. Li, "Collaborative localization with received-signal strength in wireless sensor networks," IEEE Trans. Veh. Technol., vol. 56, no. 6, pp. 3807-3817, 2007.

[5] H. Wymeersch, J. Lien, and M. Z. Win, "Cooperative localization in wireless networks," Proc. IEEE, vol. 97, no. 2, pp. 427-450, Feb. 2009, special issue on Ultra-Wide Bandwidth (UWB) Technology \& Emerging Applications.

[6] Y. Shang, W. Rumi, Y. Zhang, and M. Fromherz, "Localization from connectivity in sensor networks," IEEE Trans. Parallel Distrib. Syst., vol. 15, no. 11, pp. 961-974, Nov. 2004.

[7] X. Ji and H. Zha, "Sensor positioning in wireless ad-hoc sensor networks using multidimensional scaling," in Proc. IEEE Conf. Computer Communications (INFOCOM), vol. 4, Mar. 2004, pp. 2652-2661.
[8] J. A. Costa, N. Patwari, and A. O. Hero III, "Distributed weightedmultidimensional scaling for node localization in sensor networks," ACM Trans. Sen. Netw., vol. 2, no. 1, pp. 39-64, Feb. 2006.

[9] P. Biswas, T.-C. Lian, T.-C. Wang, and Y. Ye, "Semidefinite programming based algorithms for sensor network localization," ACM Trans. Sen. Netw., vol. 2, no. 2, pp. 188-220, May 2006.

[10] R. M. Vaghefi, M. R. Gholami, R. M. Buehrer, and E. G. Strom, "Cooperative received signal strength-based sensor localization with unknown transmit powers," IEEE Trans. Signal Process., vol. 61, no. 6, pp. 1389-1403, Mar. 2013.

[11] T. Jia and R. Buehrer, "Collaborative position location with NLOS mitigation," in Proc. IEEE Int. Symp. Personal, Indoor and Mobile Radio Communications Workshops (PIMRC Workshops), Sep. 2010, pp. 267271.

[12] T. Jia and R. M. Buehrer, "Collaborative position location for wireless networks using iterative parallel projection method," in Proc. IEEE Int. Global Telecommunications Conference (GLOBECOM), Dec 2010, pp. $1-6$.

[13] A. T. Ihler, J. W. Fisher III, R. L. Moses, and A. S. Willsky, "Nonparametric belief propagation for self-localization of sensor networks," IEEE J. Sel. Areas Commun., vol. 23, no. 4, pp. 809-819, Apr. 2005.

[14] V. Savic and S. Zazo, "Nonparametric belief propagation based on spanning trees for cooperative localization in wireless sensor networks," in Proc. IEEE Int. Conf. Veh. Technol., 2010, pp. 1-5.

[15] P.-A. Oikonomou-Filandras and K.-K. Wong, "Hybrid non-parametric belief propagation for localization in wireless networks," in Sensor Signal Processing for Defence (SSPD 2011), 2011, pp. 1-5.

[16] J. Lien, U. J. Ferner, W. Srichavengsup, H. Wymeersch, and M. Z. Win, "A comparison of parametric and sample-based message representation in cooperative localization," International Journal of Navigation and Observation, vol. 2012, pp. 1-10, 2012.

[17] K. Das and H. Wymeersch, "Censoring for bayesian cooperative positioning in dense wireless networks," IEEE J. Sel. Areas Commun., vol. 30, no. 9, pp. 1835-1842, 2012.

[18] J. Pearl, Probabilistic Reasoning in Intelligent Systems, 2nd ed. San Francisco, CA: Morgan Kaufmann, 1988.

[19] F. Kschischang, B. J. Frey, and H.-A. Loeliger, "Factor graphs and the sum-product algorithm," IEEE Trans. Inf. Theory, vol. 47, no. 2, pp. 498-519, Feb. 2001.

[20] G. Mao, B. Fidan, and B. D. O. Anderson, "Wireless sensor network localization techniques," Comput. Networks J., vol. 51, no. 10, pp. 25292553, 2007.

[21] M. Welling and J. J. Lim, "A distributed message passing algorithm for sensor localization," in Proc. Int. Conf. on Artificial neural networks. Berlin, Heidelberg: Springer-Verlag, 2007, pp. 767-775.

[22] H. Wymeersch, S. Marano, W. M. Gifford, and M. Z. Win, "A machine learning approach to ranging error mitigation for UWB localization," IEEE Trans. Commun., vol. 60, no. 6, pp. 1719-1728, 2012.

[23] V. Savic, J. Ferrer-Coll, P. Angskog, J. Chilo, P. Stenumgaard, and E. G. Larsson, "Measurement analysis and channel modeling for TOA-based ranging in tunnels," to appear in IEEE Trans. Wireless Commun., 2014.

[24] C. Fritsche and A. Klein, "On the performance of mobile terminal tracking in urban GSM networks using particle filters," in Proc. of the European Signal Processing Conference (EUSIPCO), Glasgow, Scotland, Aug. 2009, pp. 1953-1957.

[25] F. Yin, C. Fritsche, F. Gustafsson, and A. M. Zoubir, "Robust cooperative sensor network localization via the EM criterion in LOS/NLOS environments," in Proc. IEEE Int. Workshop on Signal Processing Advances in Wireless Communications (SPAWC), 2013.

[26] D. Clark and B.-N. Vo, "Convergence analysis of the Gaussian mixture PHD filter," IEEE Trans. Signal Process., vol. 55, no. 4, pp. 1204-1212, Apr. 2007.

[27] R. A. Maronna, R. D. Martin, and V. J. Yohai, Robust Statistics: Theory and Methods. Chichester, England: John Wiley \& Sons Ltd., 2006.

[28] N. Patwari and P. Agrawal, "Calibration and measurement of signal strength for sensor localization," in Localization Algorithms and Strategies for Wireless Sensor Networks, G. Mao and B. Fidan, Eds. Hershey, New York, USA: Information Science Reference, 2009.

[29] X.-L. Meng and D. B. Rubin, "Maximum likelihood estimation via the ECM algorithm: A general framework," Biometrika, vol. 80, no. 2, pp. 267-278, 1993.

[30] F. Yin, "Robust wireless localization in harsh mixed line-ofsight/non-line-of-sight environments," Ph.D. dissertation, Fachbereich Elektrotechnik und Informationstechnik, Technische Universitaet Darmstadt, Darmstadt, Germany, Aug. 2014. [Online]. Available: http://tuprints.ulb.tu-darmstadt.de/4122/ 
[31] F. Yin, C. Fritsche, F. Gustafsson, and A. M. Zoubir, "TOA-based robust wireless geolocation and Cramér-Rao lower bound analysis in harsh LOS/NLOS environments," IEEE Trans. Signal Process., vol. 61, no. 9, pp. 2243-2255, May. 2013

[32] —, "EM- and JMAP-ML based joint estimation algorithms for robust wireless geolocation in mixed LOS/NLOS environments," IEEE Trans. Signal Process., vol. 62, no. 1, pp. 168-182, Jan. 2014.

[33] A. P. Dempster, N. Laird, and D. B. Rubin, "Maximum likelihood from incomplete data via the EM algorithm," J. Roy. Statist. Soc. B, vol. 39, pp. 1-38, 1977.

[34] A. G. Dimakis, S. Kar, J. M. F. Moura, M. G. Rabbat, and A. Scaglione, "Gossip algorithms for distributed signal processing," Proc. IEEE, vol. 98, no. 11, pp. 1847-1864, 2010 .

[35] R. D. Nowak, "Distributed EM algorithms for density estimation and clustering in sensor networks," IEEE Trans. Signal Process., vol. 51, no. 8, pp. 2245-2253, Aug. 2003.

[36] M. G. Rabbat and R. D. Nowak, "Decentralized source localization and tracking wireless sensor networks," in Proc. IEEE Int. Conf. Acoustics, Speech, and Signal Processing (ICASSP), vol. 3, May 2004, pp. 921924.

[37] W. Kowalczyk and N. Vlassis, "Newscast EM," in Proc. Adv. Neural Inf. Process. Syst. Vancouver, BC, Canada: MIT Press, Dec. 2005, pp. 713-720.

[38] M. Jelasity, W. Kowalczyk, and M. Van Steen, "Newscast computing," Dept. of Computer Science, Vrije Universiteit Amsterdam, Tech. Rep., 2003.

[39] S. Boyd, A. Ghosh, B. Prabhakar, and D. Shah, "Randomized gossip algorithms," IEEE Trans. Inf. Theory, vol. 52, no. 6, pp. 2508-2530, 2006.

[40] P. Forero, A. Cano, and G. Giannakis, "Consensus-based distributed expectation-maximization algorithm for density estimation and classification using wireless sensor networks," in Proc. IEEE Int. Conf. Acoustics, Speech and Signal Processing (ICASSP), 2008, pp. 19891992.

[41] Z. J. Towfic, J.-S. Chen, and A. H. Sayed, "Collaborative learning of mixture models using diffusion adaptation," in Proc. IEEE Int Workshop on Machine Learning for Signal Processing (MLSP), 2011, pp. 1-6.

[42] R. Zekavat and R. M. Buehrer, Handbook of Position Location. Hoboken, NJ: John Wiley \& Sons, Inc., 2011.

[43] G. J. Pottie and W. J. Kaiser, "Wireless integrated network sensors," ACM Commun., vol. 43, no. 5, pp. 51-58, May 2000.

[44] M. G. Rabbat and R. D. Nowak, "Distributed optimization in sensor networks," in Proc. Int. Symp. on Information Processing in Sensor Networks (IPSN), 2004, pp. 20-27.

[45] F. Gustafsson, Statistical Sensor Fusion. Lund, Sweden: Studentlitteratur, 2012.

[46] Z. I. Botev, J. F. Grotowski, and D. P. Kroese, "Kernel density estimation via diffusion," Annals of Statistics, vol. 38, no. 5, pp. 2916-2957, 2010.

[47] G. Strang, Linear Algebra and Its Applications, 4th ed. Cengage Learning, 2005.

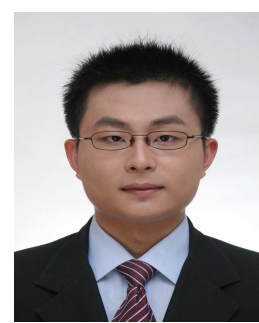

Feng Yin received his Dr.-Ing. degree from Technische Universität Darmstadt (TUD), Darmstadt, Germany in 2014. He is currently with Ericsson Research, Linköping. His research interests lie in statistical signal processing and sensor fusion for wireless localization and tracking.

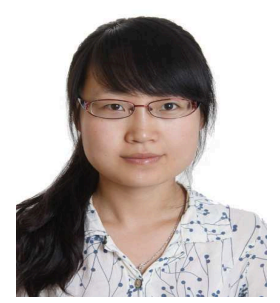

Di Jin received the B.Sc. degree in information and communication engineering from Zhejiang University, Hangzhou, China in 2011, and the M.Sc. degree in electrical engineering and information technology from Technische Universität Darmstadt, Darmstadt, Germany in 2014. She is currently working towards the Ph.D. degree in the Signal Processing Group at Technische Universität Darmstadt. Her research interests include localization and tracking, distributed and cooperative inference in wireless networks.
Carsten Fritsche received the Dipl.-Ing. degree in 2005 and the Dr.-Ing. degree in 2011 both in electrical engineering and information technology from Technische Universitt Darmstadt, Germany. In 2011 he joined the Division of Automatic Control at Linkping University, Sweden, as a post-doctoral research fellow. From 2012-2014, he was with IFEN $\mathrm{GmbH}$, Poing, Germany, working as a systems engineer and project manager on satellite navigation related applications. Since summer 2014 he has been with the Division of Automatic Control at Linkping University, Sweden. Since 2015, he additionally has been with NIRA Dynamics AB, Linkping, Sweden, working as a systems engineer in the predevelopment of next generation automotive applications. His main research interest lies in the area of statistical signal processing, machine learning and sensor fusion with applications to wireless positioning, satellite navigation and driver assistance systems.

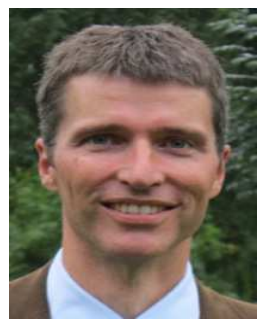

Fredrik Gustafsson is professor in Sensor Informatics at Department of Electrical Engineering, Linköping University, since 2005. He received the M.Sc. degree in electrical engineering 1988 and the $\mathrm{Ph} . \mathrm{D}$. degree in Automatic Control, 1992, both from Linköping University. During 1992-1999 he held various positions in automatic control, and 19992005 he had a professorship in Communication Systems. His research interests are in stochastic signal processing, adaptive filtering and change detection, with applications to communication, vehicular, airborne, and audio systems. He is a co-founder of the companies NIRA Dynamics (automotive safety systems), Softube (audio effects) and SenionLab (indoor positioning systems). He was an associate editor for IEEE Transactions on Signal Processing 2000-2006 and is currently associate editor for IEEE Transactions on Aerospace and Electronic Systems and EURASIP Journal on Applied Signal Processing. He was awarded the Arnberg prize by the Royal Swedish Academy of Science (KVA) 2004, elected member of the Royal Academy of Engineering Sciences (IVA) 2007, elevated to IEEE Fellow 2011 and awarded the Harry Rowe Mimno Award 2011 for the tutorial "Particle Filter Theory and Practice with Positioning Applications", which was published in the AESS Magazine in July 2010.

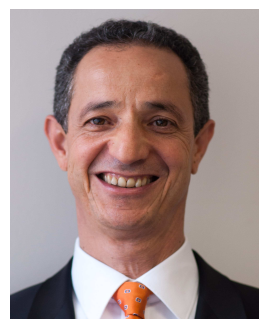

Abdelhak M. Zoubir is a Fellow of the IEEE and IEEE Distinguished Lecturer (Class 2010-2011). $\mathrm{He}$ received his Dr.-Ing. from Ruhr- Universität Bochum, Germany in 1992. He was with Queensland University of Technology, Australia from 1992-1998 where he was Associate Professor. In 1999, he joined Curtin University of Technology, Australia as a Professor of Telecommunications and was Interim Head of the School of Electrical \& Computer Engineering from 2001 until 2003. In 2003, he moved to Technische Universität Darmstadt, Germany as Professor of Signal Processing and Head of the Signal Processing Group. His research interest lies in statistical methods for signal processing with emphasis on bootstrap techniques, robust detection and estimation and array processing applied to telecommunications, radar, sonar, automotive monitoring and safety, and biomedicine. He published over 300 journal and conference papers on these areas. Professor Zoubir was the Technical Chair of the 11th IEEE Workshop on Statistical Signal Processing (SSP 2001), General Co-Chair of the 3rd IEEE International Symposium on Signal Processing \& Information Technology (ISSPIT 2003) and of the 5th IEEE Workshop on Sensor Array and Multi-channel Signal Processing (SAM 2008) and of the 14th International Workshop on Signal Processing Advances in Wireless Communications (SPAWC 2013) and of the 21st European Signal Processing Conference (EUSIPCO 2013), and the Technical Co-Chair of ICASSP-14. Dr. Zoubir was an Associate Editor of the IEEE Transactions on Signal Processing (1999-2005), a Member of the Senior Editorial Board of the IEEE Journal on Selected Topics in Signal Processing (2009-2011). He currently serves a three-year term as Editor-In-Chief of the IEEE Signal Processing Magazine (2012-2014). Dr. Zoubir was Past Chair (2012), Chair (2010-2011), Vice-Chair (2008-2009) and Member (2002-2007) of the IEEE SPS Technical Committee Signal Processing Theory and Methods (SPTM). He was a Member of the IEEE SPS Technical Committee Sensor Array and Multi-channel Signal Processing (SAM) from 2007 until 2012. He also serves on the Board of Directors of the European Association of Signal Processing (EURASIP). 